\title{
Simple Finite Element Numerical Simulation of Incompressible Flow Over Non-rectangular Domains and the Super-Convergence Analysis
}

\author{
Yunhua Xue $^{1}$ - Cheng Wang ${ }^{2} \cdot$ Jian-Guo Liu ${ }^{3}$ \\ Received: 11 May 2014 / Accepted: 5 March 2015 / Revised: 10 February 2015 \\ Published online: 14 March 2015 \\ (C) Springer Science+Business Media New York 2015
}

\begin{abstract}
In this paper, we apply a simple finite element numerical scheme, proposed in an earlier work (Liu in Math Comput 70(234):579-593, 2000), to perform a high resolution numerical simulation of incompressible flow over an irregular domain and analyze its boundary layer separation. Compared with many classical finite element fluid solvers, this numerical method avoids a Stokes solver, and only two Poisson-like equations need to be solved at each time step/stage. In addition, its combination with the fully explicit fourth order Runge-Kutta (RK4) time discretization enables us to compute high Reynolds number flow in a very efficient way. As an application of this robust numerical solver, the dynamical mechanism of the boundary layer separation for a triangular cavity flow with Reynolds numbers $R e=10^{4}$ and $R e=10^{5}$, including the precise values of bifurcation location and critical time, are reported in this paper. In addition, we provide a super-convergence analysis for the simple finite element numerical scheme, using linear elements over a uniform triangulation with right triangles.
\end{abstract}

Keywords Incompressible flows - Boundary layer separation - Structural bifurcation . Simple finite element method · Super-convergence analysis

Mathematics Subject Classification $65 \mathrm{~N} 30 \cdot 65 \mathrm{M} 12 \cdot 35 \mathrm{Q} 30 \cdot 76 \mathrm{D} 05$

$\triangle \quad$ Cheng Wang

cwang1@umassd.edu

Yunhua Xue

yhxue@nankai.edu.cn

Jian-Guo Liu

jian-guo.liu@duke.edu

1 School of Mathematics Sciences and LPMC, Nankai University, Tianjin 300071, China

2 Department of Mathematics, University of Massachusetts Dartmouth, North Dartmouth, MA 02747-2300, USA

3 Department of Physics and Department of Mathematics, Duke University, Durham, NC 27708, USA 


\section{Introduction}

The primary goal of this paper is to numerically investigate incompressible flow over non-rectangular domains, with no-penetration, no-slip boundary condition, and provide a super-convergence analysis for the simple finite element scheme using linear element. The vorticity-stream function formulation of two-dimensional (2-D) incompressible NavierStokes equations (NSE) is considered, which reads,

$$
\begin{aligned}
\partial_{t} \omega+(\boldsymbol{u} \cdot \nabla) \omega & =v \Delta \omega+f, \quad \text { in } \Omega, \\
\Delta \psi & =\omega, \quad \text { in } \Omega, \\
\boldsymbol{u} & =\nabla^{\perp} \psi:=\left(-\partial_{y} \psi, \partial_{x} \psi\right),
\end{aligned}
$$

and the no-penetration, no-slip boundary condition is formulated in terms of the stream function:

$$
\psi=0, \quad \frac{\partial \psi}{\partial \boldsymbol{n}}=0, \quad \text { on } \partial \Omega .
$$

Here $\Omega$ is assumed to be a bounded, simply connected domain with Lipschitz continuous boundary $\Gamma=\partial \Omega$, and we only consider the 2 -D polygon domain in this paper. The Reynolds number is defined as $R e=\frac{L U}{v}$, in which $L, U$ and $v$ denote the length scale, velocity scale and the kinematic viscosity, respectively. For simplicity, we take $L=1, U=1$ so that $R e=\frac{1}{v}$ in this paper.

It is well-known that a boundary layer appears in any viscous incompressible fluid, due to the slow-down of the flow by a no-slip boundary $[26,44]$. Moreover, this shear flow may detach and separate from the boundary, generating vorticity and a recirculation area for flow with a high Reynolds number.

Many experimental observations have indicated that the point where the vorticity vanishes on the boundary may be a candidate for separation of the boundary layer; see the descriptions in [6]. The theoretical justification of this fact has been provided in a few works [21-23], using an orbit analysis approach for 2-D divergence-free vector field. In more detail, the structural bifurcation on the boundary is associated with the boundary layer separation, and such a bifurcation is assured to occur when the vorticity reaches zero value as a local maximum (minimum), with a positive (negative) time derivative.

The qualitative description of the structural bifurcation of incompressible NSE is valid for any domain, either rectangular or non-rectangular. Obviously, such a study on an irregular, non-rectangular domain would lead to plenty of practical applications, such as flow past an obstacle, oceanic flow, blood flow in the vessel, etc.

There have been many earlier numerical works for high Reynolds number flow, such as the ones over a rectangular domain using finite difference or spectral methods $[5,11-$ $14,17,20,34,35]$, the flow past cylinder [36,37], etc. Also see the related works $[18,19,32,41]$ using either a curvilinear domain or an immersed boundary method. In these numerical simulations, the computational domain is either rectangular or could be conformally mapped to a rectangular domain, so that a fast Poisson solver could be easily obtained, which in turn leads to a great numerical efficiency. Subsequently, a natural question arises: how to perform a numerical simulation of a high Reynolds number flow over an irregular domain in an efficient way so that the boundary layer separation could be studied in detail?

The finite element method is especially suitable for the numerical simulation over an irregular domain, due to its domain flexibility; see the related references [2,8-10,24, 25,28$31,39,46,48,49]$, etc. Meanwhile, it is observed that most of these existing works are for 
stationary flow or nonstationary flow with moderate Reynolds number $\left(\operatorname{Re} \leq 10^{3}\right.$ ); no detailed numerical exploration for the boundary layer separation over a non-rectangular domain has been reported. The key reason is that, most existing finite element works for incompressible fluid are based on a Stokes solver, in which the divergence-free condition and the pressure gradient are coupled together. As a result of this highly non-trivial coupling, a well-resolved numerical simulation of boundary layer separation becomes computationally costly and very challenging.

In this article, we apply a simple finite element method for (1)-(3), proposed by E \& Liu [43], to the numerical study of boundary layer separation over a triangular domain. The simple means that $k$-th $(k=2,3)$ order finite elements can be used for both stream function and vorticity. This finite element scheme avoids a Stokes solver, and only two Poisson/Poissonlike equations need to be solved at each time step/stage. This approach greatly improves the computational efficiency for high Reynolds number flow, in conjunction with a fully explicit Runge-Kutta (RK4) time discretization. The numerical stability of this fully explicit time stepping follows the linearized stability domain argument; see the related discussions about the finite difference approximation for high Reynolds flow [11,12], where the convection and viscous terms are both treated explicitly in the vorticity transport equation.

The numerical scheme using a linear element is applied to the study of the structure bifurcation of flow and boundary layer separation over a right triangle domain. The linear element approximation shows a great numerical advantage, since it usually leads to a smaller band width of stiff matrix, compared with the higher order elements. There are many existing numerical works for a triangular cavity flow, see the related references $[1,14,27,33,38,40$, 47], etc. Again, due to the numerical complexity for high Reynolds number flows, no report on the boundary layer separation has been presented in these works. In our work, we demonstrate the detailed transition process for the structural bifurcation on the triangular domain.

In addition, we provide an improved convergence analysis for the simple finite element presented in [43]. In the original article, the convergence order is given by $h^{k-1 / 2}$, with $k$ the polynomial degree in the finite element space. However, a careful observation indicates that the presented convergence analysis is only valid for the elements with $k \geq 2$, such as the quadratic $(k=2)$ and cubic $(k=3)$ polynomials. An analysis of the linear element scheme, which is of great practical interests due to its smaller band width, has not been justified at the theoretical level. The reason for this subtle fact is that, the $L^{\infty}\left(0, T ; L^{2}\right)$ error estimate for the velocity variable yields an $O\left(h^{k-1 / 2}\right)=O\left(h^{1 / 2}\right)$ (with $k=1$ ) convergence, and an application of standard inverse inequality fails to recover the $L^{\infty}$ bound of the numerical solution for the velocity, in both 2-D and 3-D cases. This $L^{\infty}$ recovery has always played a crucial role in the nonlinear error estimate.

In this paper, we present a super-convergence analysis of the simple finite element scheme using a linear element over a uniform triangulation $\mathcal{T}_{h}$ with right triangle cells. To overcome the above mentioned difficulties, we apply the super-convergent property of a uniform triangulation so that the accuracy could be improved by $h^{1 / 2}$; see the related analysis $[42,50]$. In more detail, with this super-convergence feature, the $L^{\infty}\left(0, T ; L^{2}\right)$ convergence order for the velocity variable, in conjunction with the $L^{2}\left(0, T ; L^{2}\right)$ convergence order for the vorticity variable, is improved to $O(h)$, under an a-priori $L^{2}\left(0, T ; L^{\infty}\right)$ bound of the numerical solution for the velocity variable. In turn, the rest work is focused on the recovery of such an a-priori $L^{2}\left(0, T ; L^{\infty}\right)$ bound for the velocity. First, we make use of the $O(h)$ convergence order for the vorticity variable, in the $L^{2}\left(0, T ; L^{2}\right)$ norm. Next, a $W^{1,4}$ estimate of the standard finite element Poisson solver is recalled, which yields an $O(h)$ error estimate for the velocity variable in the $L^{2}\left(0, T ; L^{4}\right)$ norm. Subsequently, an application of an alternate inverse inequality results in an $O\left(h^{1 / 2}\right)$ estimate for the $L^{2}\left(0, T ; L^{\infty}\right)$ error of the velocity 
variable, and this becomes a uniform bound over a given final time. Therefore, the a-priori $L^{2}\left(0, T ; L^{\infty}\right)$ bound for the velocity is justified by the triangle inequality, and the nonlinear error analysis could go through.

The remaining part of this paper is organized as follows. The simple finite element method is recalled in Sect. 2. In Sect. 3, the simple finite element scheme is applied to a triangular cavity flow and the numerical investigation of the boundary layer separation is presented in detail. In Sect. 4, we provide a detailed error estimate and super-convergence analysis for the linear element scheme, using a uniform triangulation with right triangle cells, and the convergence rate is given by $O(h)$ for both the $L^{\infty}\left(0, T ; L^{2}\right)$ error for the velocity variable and the $L^{2}\left(0, T ; L^{2}\right)$ error for the vorticity variable. A numerical example of accuracy check over a trapezoid domain is presented in Sect. 5, with the super-convergence result observed. Finally, some concluding remarks are made in Sect. 6.

\section{Review of Simple Finite Element Discretization and the Convergence Order}

Some usual notations are used in the finite element framework. Let $H^{r}(\Omega), r \geq 0$ be the standard Sobolev space on the domain $\Omega$, associated with the norm $\|\cdot\|_{r, \Omega}$ and the semi-norm $|\cdot|_{r, \Omega}$. In case $r=0$, we have $H^{0}(\Omega)=L^{2}(\Omega)$. For the function $\phi(\boldsymbol{x}, t)$, which depends on both the spatial variable $\boldsymbol{x}$ and the temporal variable $t$, the norm $\|\phi(\boldsymbol{x}, t)\|_{L^{p}\left((0, T] ; H^{r}(\Omega)\right)}$ is defined as $\|\Phi(t)\|_{L^{p}((0, T])}$, with $\Phi(t)=\|\phi(\boldsymbol{x}, t)\|_{H^{r}(\Omega)}$. For the $n$-dimensional vector functions with components in one of these spaces, we shall use the notation

$$
\begin{aligned}
& \boldsymbol{L}^{p}(\Omega)=\left\{L^{p}(\Omega)\right\}^{n}, \boldsymbol{H}^{r}(\Omega)=\left\{H^{r}(\Omega)\right\}^{n}, \\
& \boldsymbol{L}^{p}\left((0, T] ; \boldsymbol{H}^{r}(\Omega)\right)=\left\{L^{p}\left((0, T] ; H^{r}(\Omega)\right)\right\}^{n} .
\end{aligned}
$$

For simplicity, we assume that $\Omega$ is a polygonal domain. Let $\mathcal{T}_{h}$ be a quasi-uniform triangulation of $\Omega$, and the mesh parameter is given by $h=\max _{K \in \mathcal{T}_{h}} h_{K}$, where $h_{K}$ stands for the diameter of triangle element $K$. And also, $X_{h}^{k}$ be the standard continuous finite element space with $k$-th degree polynomials on each element of $\mathcal{T}_{h}$. Denote $X_{0, h}^{k}$ be the subspace of $X_{h}^{k}$ with zero boundary values.

The NSE (1)-(3) can be formulated in a weak form: find $\omega \in H^{1}(\Omega)$ and $\psi \in H_{0}^{1}(\Omega)$ such that

$$
\begin{aligned}
\left(\phi, \partial_{t} \omega\right)-(\nabla \phi, \omega \boldsymbol{u}) & =-v(\nabla \phi, \nabla \omega)+(f, \phi), \quad \forall \phi \in H_{0}^{1}(\Omega), \\
(\nabla \phi, \nabla \psi) & =-(\phi, \omega), \quad \forall \phi \in H^{1}(\Omega) .
\end{aligned}
$$

See [43] for a detailed derivation.

In turn, the finite element semi-discretization scheme is given as follows: find $\omega_{h} \in X_{h}^{k}$ and $\psi_{h} \in X_{0, h}^{k}$, such that

$$
\begin{aligned}
\left(\phi, \partial_{t} \omega_{h}\right)-\left(\nabla \phi, \omega_{h} \boldsymbol{u}_{h}\right) & =-v\left(\nabla \phi, \nabla \omega_{h}\right)+(f, \phi), \quad \forall \phi \in X_{0, h}^{k}, \\
\left(\nabla \phi, \nabla \psi_{h}\right) & =-\left(\phi, \omega_{h}\right), \quad \forall \phi \in X_{h}^{k} .
\end{aligned}
$$

In addition, we denote the numerical velocity as $\boldsymbol{u}_{h}=\nabla^{\perp} \psi$.

For the time stepping procedure to the semi-discretization formulation $(9,10)$, we use the classical RK4 in the computation, since its stability region encompasses an appreciable portion of the imaginary axis. In the RK4 time stepping procedure, computations of the vorticity and stream function have been fully decoupled, so that a Stokes solver is avoided. 
No iteration is required between the vorticity and stream function to recover the bi-harmonic operator. The main computation is involved with solving a standard Poisson equation and inverting a standard mass matrix. Among these two linear systems, most computational costs will be paid for the Poisson solver, due to an $O(1)$ condition number of the mass matrix. To implement the simple finite element scheme, we use Hypre solver package (High performance preconditioners) for the parallel codes [16].

In addition, a purely explicit treatment of the nonlinear term also greatly simplifies the computational effort. As for the time step constraint, the fully discrete scheme (in conjunction with the RK4 time stepping) is stable as long as $\Delta t$ satisfy the following two conditions:

$$
\left\|\boldsymbol{u}_{h}\right\|_{\boldsymbol{L}^{\infty}\left((0, T] ; \boldsymbol{L}^{\infty}(\Omega)\right)} \frac{\triangle t}{h}<C_{1}, \quad 4 v \frac{\Delta t}{h^{2}}<C_{2} .
$$

The detailed description for this choice is referred to [12], in which the finite difference approximation was taken as the spatial discretization.

More importantly, the Ladyzhenskaya-Babuska-Brezzi (LBB) condition is not required in the finite element space construction, compared with the Stokes solver-based finite element schemes for incompressible fluid [3,8,24,25,29,30,39,49]. The detailed description has been outlined in [43]. This fact greatly improves the computational efficiency for high Reynolds number flow, as demonstrated by the numerical examples presented in Sect. 3.

Remark 2.1 In addition to the classical RK4, some other multi-stage RK schemes can also be used. Meanwhile, for the computation of moderate to high Reynolds number flows, the corresponding RK scheme has to have stability region that encompasses an appreciable portion of the imaginary axis. From this point of view, both RK3 and RK4 are good choices, while RK2 is insufficient for moderate to high Reynolds number flows since its stability region does not contain any portion of the imaginary axis.

In addition, the following convergence result was provided in [43], using mixed finite element analysis.

Theorem 2.1 [43] Let $(\psi, \omega, \boldsymbol{u})$ satisfy $(7,8)$ and $\left(\psi_{h}, \omega_{h}, \boldsymbol{u}_{h}\right)$ be the numerical solution of $(9,10)$ with $k$-th order finite element space $X_{h}^{k},(k \geq 2)$. Then we have

$$
\left\|\boldsymbol{u}-\boldsymbol{u}_{h}\right\|_{\boldsymbol{L}^{\infty}\left((0, T] ; \boldsymbol{L}^{2}(\Omega)\right)}+\left\|\omega-\omega_{h}\right\|_{L^{2}\left((0, T] ; L^{2}(\Omega)\right)} \leq C h^{k-1 / 2},
$$

where $C$ is a constant only dependent on the exact solution, the final time $T$, and the kinematic viscosity $v$, independent of $h$.

In the above theorem, higher order finite element $(k \geq 2)$ have to be used to ensure the convergence order. In Sect. 4, an improved convergence result is shown to be valid for the linear element scheme, and a super-convergence will be proven.

\section{Numerical Simulation of the Structure Bifurcation and Boundary Layer Separation}

The general theory of structural stability and bifurcation for 2-D divergence-free vector fields is referred to [21-23]. For simplicity, we only outline the main result for structural bifurcation associated with the boundary layer separation. 
Denote $D^{r}(\Omega)$ and $B_{0}^{r}(\Omega)$ as

$$
\begin{aligned}
& D^{r}(\Omega)=\left\{\boldsymbol{u} \in C_{n}^{r}(\Omega) \quad\left|\boldsymbol{u}_{n}\right|_{\partial \Omega}=0, \operatorname{div} \boldsymbol{u}=0\right\}, \\
& B_{0}^{r}(\Omega)=\left\{\boldsymbol{u} \in D^{r}(\Omega) \quad|\boldsymbol{u}|_{\partial \Omega}=0\right\},
\end{aligned}
$$

where $\boldsymbol{u}_{n}=\boldsymbol{u} \cdot \boldsymbol{n}$ and $\boldsymbol{u}_{\tau}=\boldsymbol{u} \cdot \boldsymbol{\tau}, \boldsymbol{n}$ and $\boldsymbol{\tau}$ are the unit normal and tangent vectors on $\partial \Omega$, respectively. A point $P \in \Omega$ is called a singular point of $\boldsymbol{v}$ if $\boldsymbol{v}(P)=0$. A point $P \in \partial \Omega$ is called a $\partial$-regular point of $\boldsymbol{v}$ if $\partial \boldsymbol{v}_{\tau}(P) / \partial \boldsymbol{n} \neq 0$; otherwise, $P \in \partial \Omega$ is called a $\partial$-singular point of $\boldsymbol{v}$.

Let $\boldsymbol{u}, \boldsymbol{v} \in C^{1}\left([0, T], B_{0}^{r}(\Omega)\right)$ have the following Taylor expansions:

$$
\begin{aligned}
\boldsymbol{u}(\boldsymbol{x}, t) & =\boldsymbol{u}^{0}(\boldsymbol{x})+\left(t-t_{0}\right) \boldsymbol{u}^{1}(\boldsymbol{x})+O\left(\left|t-t_{0}\right|^{2}\right) \\
\boldsymbol{v}(\boldsymbol{x}, t) & =\boldsymbol{v}^{0}(\boldsymbol{x})+\left(t-t_{0}\right) \boldsymbol{v}^{1}(\boldsymbol{x})+O\left(\left|t-t_{0}\right|^{2}\right) \\
\boldsymbol{u}^{0}(\boldsymbol{x}) & =\boldsymbol{u}\left(\boldsymbol{x}, t_{0}\right), \quad \boldsymbol{u}^{1}(\boldsymbol{x})=\frac{\partial \boldsymbol{u}\left(\boldsymbol{x}, t_{0}\right)}{\partial t} \\
\boldsymbol{v}^{0}(\boldsymbol{x}) & =\frac{\partial \boldsymbol{u}^{0}}{\partial \boldsymbol{n}}, \quad \boldsymbol{v}^{1}(\boldsymbol{x})=\frac{\partial \boldsymbol{u}^{1}}{\partial \boldsymbol{n}}
\end{aligned}
$$

in which $\boldsymbol{v}^{0}, \boldsymbol{v}^{1}$ denote the vector field associated with the normal derivative of $\boldsymbol{u}^{0}$ and $\boldsymbol{u}^{1}$, respectively.

The structural bifurcation theory is recalled and its connection with the boundary layer separation is given.

Theorem 3.1 [21-23] Let $\boldsymbol{u} \in C^{1}\left([0, T] ; B_{0}^{r}(\Omega)\right), r \geq 2$ satisfies the following assumption (with $\omega=\nabla \times \boldsymbol{u}=-u_{y}+v_{x}$ ):

$$
\omega\left(P^{*}, T^{*}\right)=0, \frac{\partial \omega}{\partial \tau}\left(P^{*}, T^{*}\right)=0, \frac{\partial^{2} \omega}{\partial \tau^{2}}\left(P^{*}, T^{*}\right) \neq 0, \frac{\partial \omega}{\partial t}\left(P^{*}, T^{*}\right) \neq 0,
$$

then we have

1. $\boldsymbol{u}(\boldsymbol{x}, t)$ has a bifurcation in its local structure at $\left(P^{*}, T^{*}\right)$; and

2. if $P^{*} \in \partial \Omega$ is a unique singular point on $\partial \Omega$, then $\boldsymbol{u}(\boldsymbol{x}, t)$ has a bifurcation in its global structure at $t=T^{*}$.

For the 2-D incompressible NSE in the velocity-pressure formulation

$$
\begin{aligned}
\partial_{t} \boldsymbol{u}+\boldsymbol{u} \cdot \nabla \boldsymbol{u}+\nabla p & =\frac{1}{R e} \Delta \boldsymbol{u}+\boldsymbol{f}, \\
\nabla \cdot \boldsymbol{u} & =0, \\
\boldsymbol{u} & =0, \quad \text { on } \partial \Omega,
\end{aligned}
$$

the structural bifurcation theory can be applied to this divergence-free vector fields $\boldsymbol{u}(t)$.

Remark 3.1 [21] The conditions on vorticity in (19) indicate that the formation of recirculating cells starts at the moment when the vorticity reaches its zero point on the boundary as a local minimum (maximum) point in space. We denote this separation point as $P^{*} \in \partial \Omega$ at critical moment $T^{*}$. When the flow is upward, this amounts mathematically to assuming that there exists a neighborhood $U_{1}$ of $P^{*}$ such that the boundary of $U_{1}$ includes a portion of $\partial \Omega$ and

$$
\omega \leq 0 \text {, in } U_{1} \text {, when } t<T^{*} \text {. }
$$


This condition implies that the vorticity reaches zero at $\left(P^{*}, T^{*}\right)$ as a local maximum in space and increases afterward in time, and vice versa. In this case, the formation of a recirculating flow region is given by the following conditions:

$$
\omega\left(P^{*}, T^{*}\right)=0, \frac{\partial \omega}{\partial \tau}\left(P^{*}, T^{*}\right)=0, \frac{\partial^{2} \omega}{\partial \tau^{2}}\left(P^{*}, T^{*}\right)<0, \frac{\partial \omega}{\partial t}\left(P^{*}, T^{*}\right)>0 .
$$

Remark 3.2 Theorem 3.1 is valid for any domain, either rectangular or non-rectangular. It has been numerically verified on the rectangular domain in [21], using a fourth order finite difference method for a square cavity flow. In this paper, we present a numerical simulation of a triangle cavity flow, in particular for its dynamical mechanism of boundary layer separation, using the simple finite element scheme. The numerical results are expected to be in a nice agreement with Theorem 3.1 and Remark 3.1.

The 2-D incompressible flow in a non-dimensional right triangle domain $\Omega$ is investigated in detail, with the boundary sections given by one vertical line $x=1$, one horizontal line $y=1$ and one oblique line $x+y=1$. Let $\Gamma_{1}$ denote the top (lid) boundary, and $\Gamma_{2}=\partial \Omega \backslash \Gamma_{1}$.

We perform the numerical simulations with two different set-ups of initial data and boundary condition. The first is a smoothly started flow, and a parabolic driven velocity is imposed on the top boundary. Since the corner singularity is avoided, this numerical approach enables us to compute the flow at a high Reynolds number in an efficient way. As a result, we present the numerical results for Reynolds numbers $R e=10^{4}$ and $R e=10^{5}$, and the detailed mechanism of boundary layer separation is illustrated through this simulation. The second one is a more physics-relevant benchmark problem: an impulsively started flow with a constant driven velocity $u \equiv 1$ on the top boundary. Due to the corner singularity, this numerical work is more challenging, and we present the results of impulsively started flow with $R e=10^{4}$.

Remark 3.3 Physical boundary layer separation is one of the most important and difficult problems in fluid dynamics. Moreover, various numerical and experimental evidences have shown that, a 3-D effect has to be taken into consideration for the high Reynolds number flows. For example, it was reported in a recent article [27] by Gonzalez et al. that the steady triangular flow is unstable to 3-D perturbations for a Reynolds number large enough.

In this paper, we do not intend to solve these fundamental problems, since they are far beyond the current computational capacity. Meanwhile, the incompressible NSE is universally accepted as an accurate model of the practical fluid problems. The example we provide is the boundary layer analysis for the incompressible NSE and itself is of great mathematical and physical interests. In this regard, the 2-D computation and the smooth initial data set-up are taken. This also gives a challenging benchmark computation. The cavity flow benchmark indeed has been a great guideline for the numerical scheme development of incompressible flow in the last three decades or more.

\subsection{Numerical Simulation of the Boundary Layer Separation over a Triangle Domain, Smoothly Started Flow}

The initial data for the stream function and vorticity, for the smoothly started, lid driven cavity flow are given by

$$
\psi_{0}(x, y)=-16(x-1)^{2}(y-1)(x+y-1)^{2}, \quad \omega_{0}(x, y)=\Delta \psi_{0}(x, y),
$$

so that the no-penetration, no-slip boundary conditions are imposed on $\Gamma_{2}$ and a nonhomogeneous Neumann boundary condition (with a parabolic slip velocity profile) is imposed on the top boundary: 


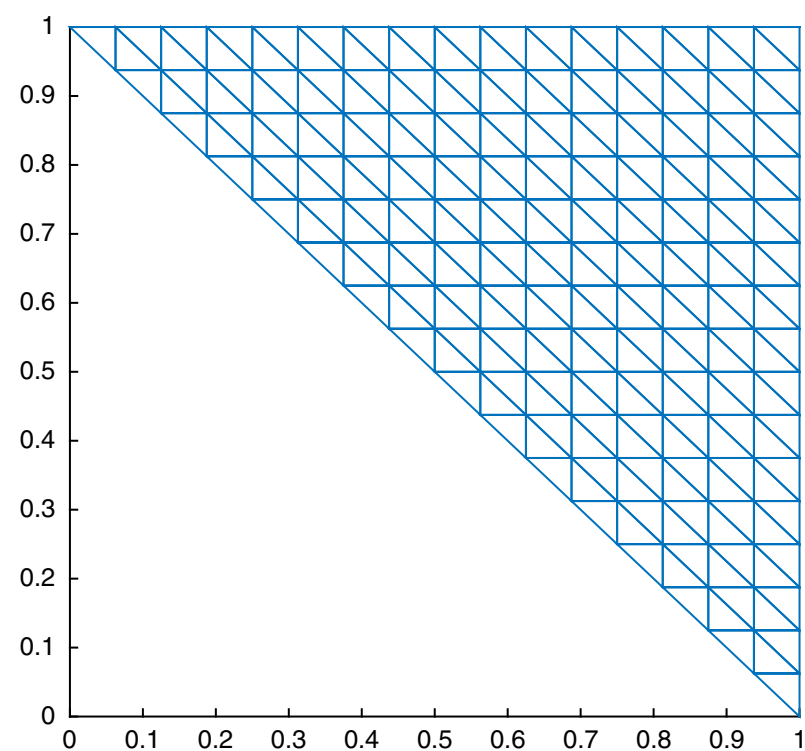

Fig. 1 The triangular domain mesh

$$
\left.\frac{\partial \psi}{\partial \boldsymbol{n}}\right|_{\Gamma_{1}}=\left.\boldsymbol{u} \cdot \boldsymbol{\tau}\right|_{\Gamma_{1}}=16(x-1)^{2} x^{2},\left.\quad \frac{\partial \psi}{\partial \boldsymbol{n}}\right|_{\Gamma_{2}}=0 .
$$

Note that these Neumann boundary conditions have to be taken into consideration in the computation of vorticity in the RK4 time stepping.

The triangular domain mesh is illustrated in Fig. 1. For all the contour plots displayed in the following sections, solid line corresponds to positive values, while dotted line corresponds to negative values, and the values in legend are their abstract values.

\subsubsection{Example 1: Flow with Reynolds number $\mathrm{Re}=10^{4}$}

To capture the detailed structures in the process of structural transition, we perform the numerical test (using the linear finite element) on a uniform triangulation $\mathcal{T}_{h}$ of $\Omega$, with mesh parameter $h=1 / 1024$. Due to the stability condition (11), we set the time step as $\Delta t=2.0 E-4$.

The structural transition condition outlined in Theorem 3.1 could be applied to the oblique line $x+y=1$, since a no-slip boundary condition is imposed there. We focus our study of the boundary layer separation mechanism on this oblique boundary section.

The numerical simulation shows that the vorticity along the upper-portion of the oblique boundary remains negative for a while after the initial time. See Fig. 2 at time $t=1.3$ for the vorticity zoomed contours and its plot on the oblique line.

During this time interval, the boundary layer structure is standard and its regularity persists until the boundary layer separation occurs. The stream function and zoomed contours at $t=1.3$, presented in Fig. 3, also clarify this standard structure.

Meanwhile, we also notice a small recirculation on the right vertical boundary $x=1$; this corresponds to another structural transition process, before the beginning of transition on the oblique boundary. As time goes on, at $t=1.8$, Fig. 4 presents the zoomed vorticity and its 

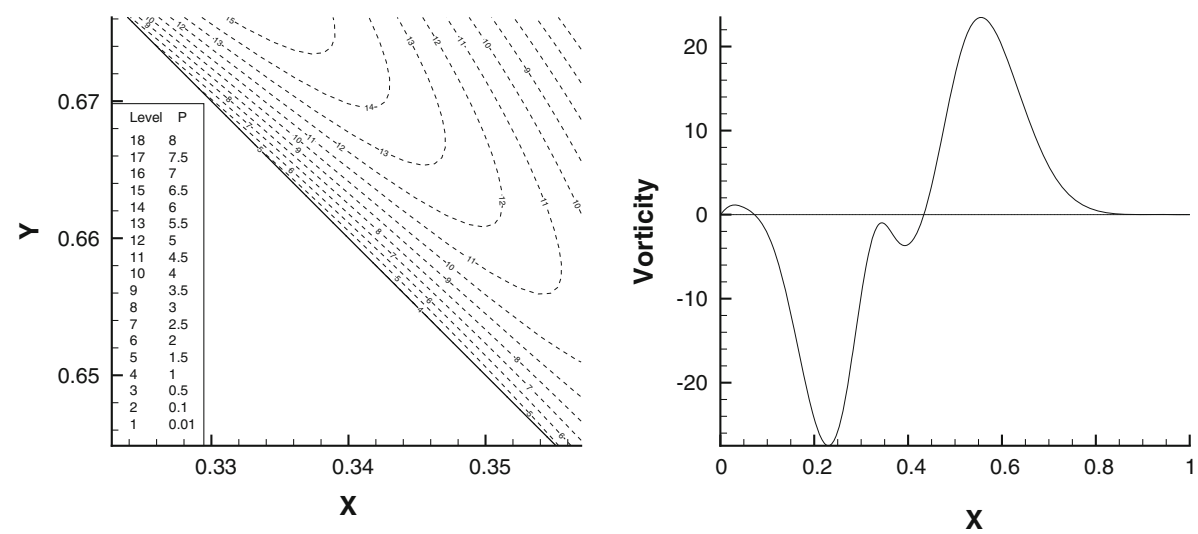

Fig. 2 The triangle cavity flow at time $t=1.3$ with $h=1 / 1024$. Left vorticity zoomed contours. Right vorticity plot on the oblique line $x+y=1$
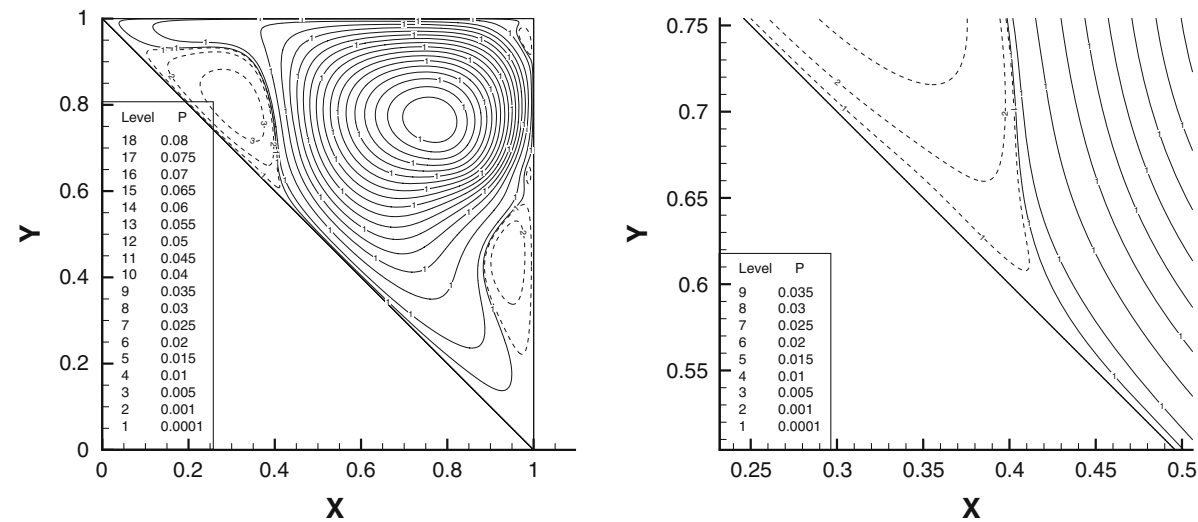

Fig. 3 The triangle cavity flow at time $t=1.3$ with $h=1 / 1024$. Left stream function contours. Right zoomed contours

plot on the oblique boundary. Similarly, the stream function and its zoomed contour plots are displayed in Fig. 5.

A clear difference in the flow structure between $t=1.3$ and $t=1.8$ can be observed by a comparison between Figs. 3, 5 for the stream function, Figs. 2, 4 for the vorticity. At $t=1.8$, the recirculation is apparent in the zoomed contour plots of stream function in Fig. 5, while such a structure does not emerge at $t=1.3$. It is also observed that the local maximum of vorticity varies from a negative value to a positive one, from $t=1.3$ to $t=1.8$. This fact is consistent with the extended discussion in Remark 3.1, since the basic recirculation is upward along the oblique boundary.

Therefore, a conclusion from Theorem 3.1 implies that the transition occurs between $t=$ 1.3 and $t=1.8$. The critical point $P^{*}$ and the critical time $T^{*}$ associated with the boundary layer separation can be numerically captured. A careful numerical experiment gives the critical time $T^{*}=1.3456$; at this time, vorticity reaches zero at $P^{*}(0.34277340,0.65722660)$, as a local maximum, see Figs. 6 and 7. The vorticity at $P^{*}$ increases along the time, so $\partial \omega / \partial t>0$. This exactly satisfies the conditions in Remark 3.1. 

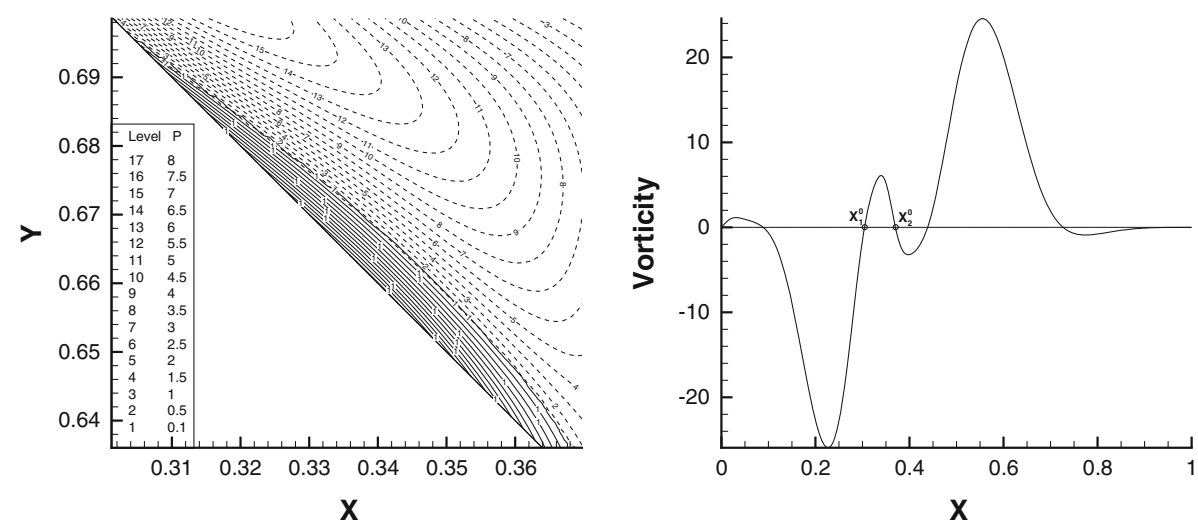

Fig. 4 The triangle cavity flow at time $t=1.8$ with $h=1 / 1024$. Left vorticity zoomed contours. Right vorticity on the oblique line $x+y=1$
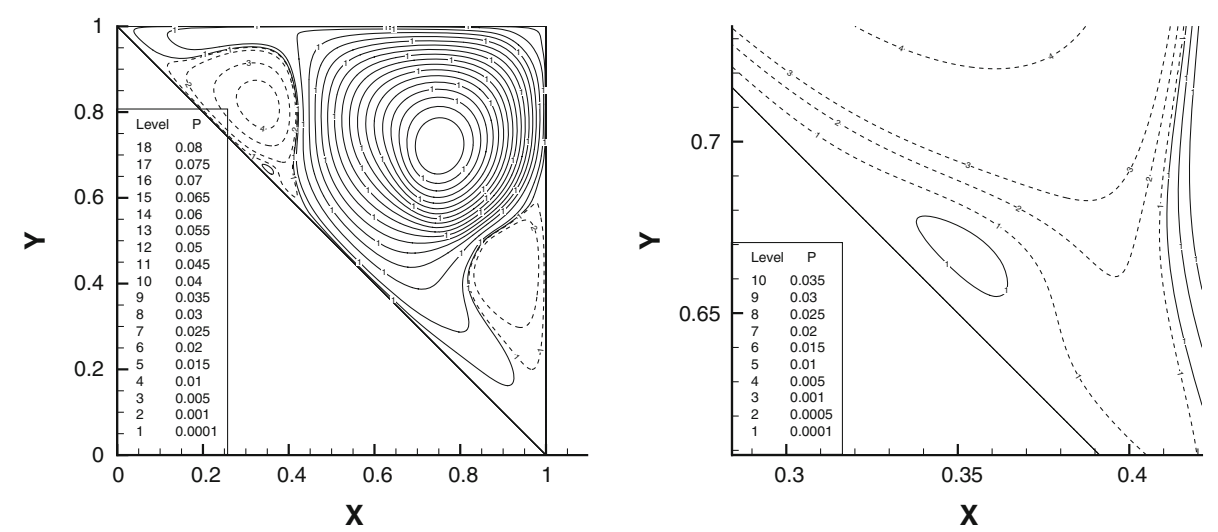

Fig. 5 The triangle cavity flow at time $t=1.8$ with $h=1 / 1024$. Left stream function contours. Right stream function zoomed contours
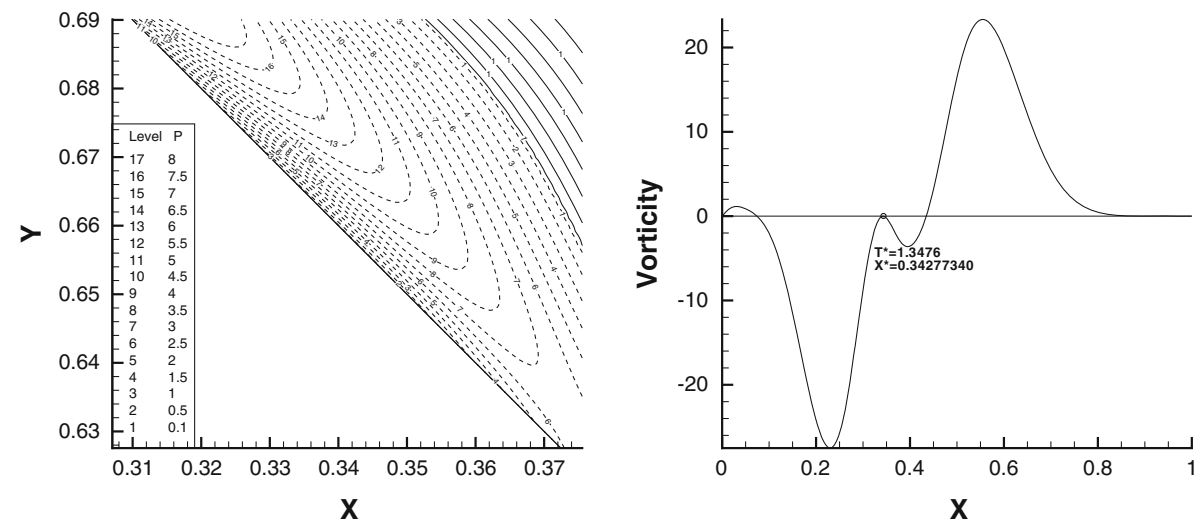

Fig. 6 The triangle cavity flow at time $t=1.3476$ with $h=1 / 1024$. Left vorticity zoomed contours. Right vorticity on the oblique line $x+y=1$ 

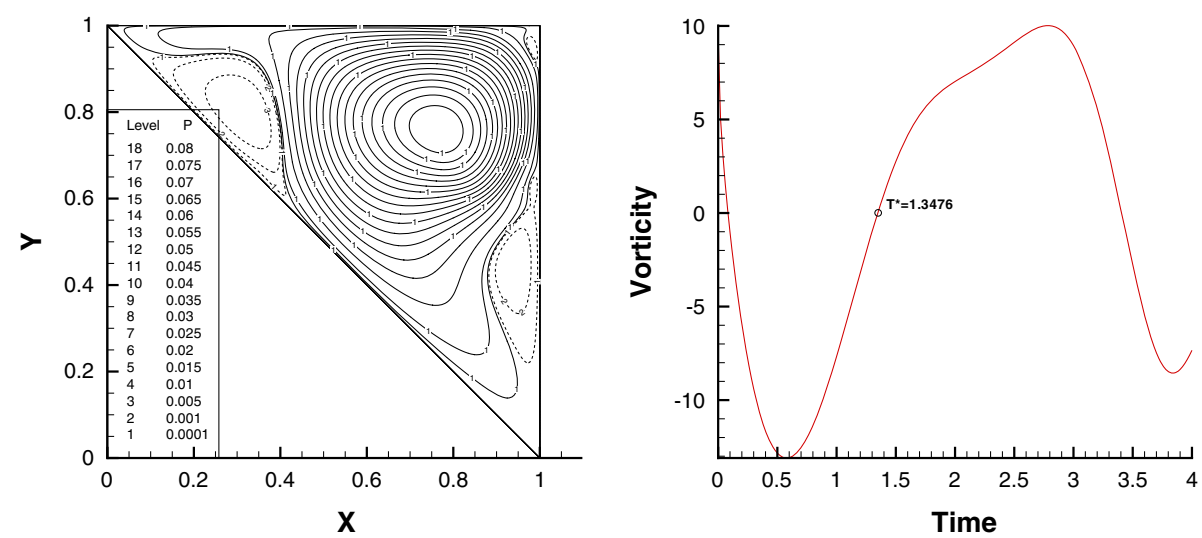

Fig. 7 The triangle cavity flow at time $t=1.3476$ with $h=1 / 1024$. Left stream function contours. Right vorticity evolves with time, at the critical point $P^{*}=(0.34277340,0.65722660)$
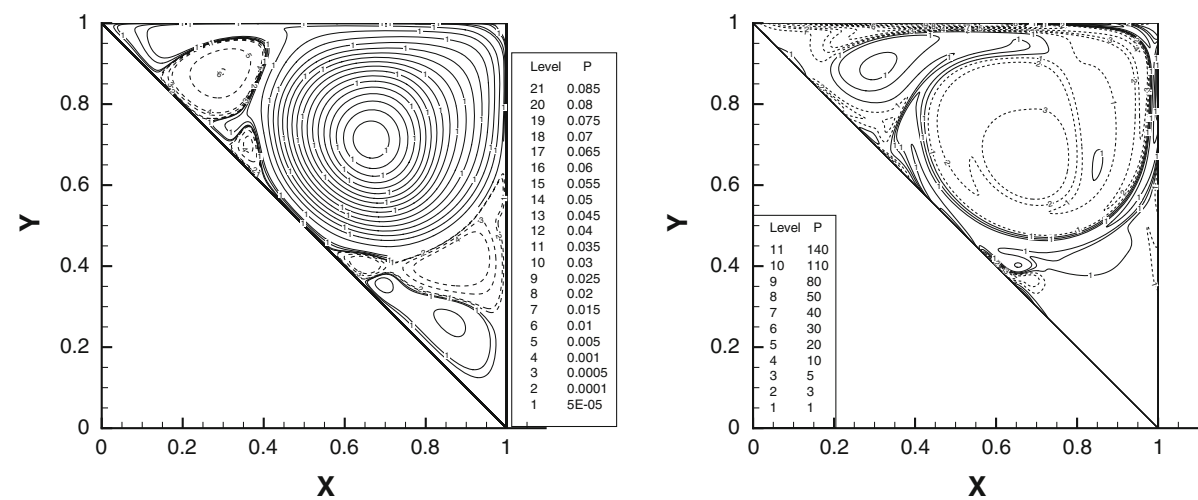

Fig. 8 The triangle cavity flow at time $t=4.0$ with $h=1 / 1024$. Left stream function contours. Right vorticity contours

At later time instants, other recirculation areas form along the boundary. We omit the detailed descriptions, which are similar to the first transition process. The contour plots of the stream function and vorticity at $t=4.0$ are displayed in Fig. 8.

\subsubsection{Double Resolution Check}

The numerical simulation of high Reynolds number fluid is highly challenging, because of many small structures of vortex roll-up, either around the boundary or at the interior region. To demonstrate the numerical accuracy for the triangular driven cavity flow, for which the explicit form of exact analytic solution is not available, we carry out the computations by using three mesh grid and time step sizes: $h=\frac{1}{384}, \Delta t=4.0 E-04$ for the 2nd order element, $h=\frac{1}{1024}, \Delta t=4.0 E-04$ for the linear element, and $h=\frac{1}{1024}, \Delta t=2.0 E-04$ for the linear element, respectively. For accuracy verification, the stream function and vorticity profiles computed by the three resolutions are compared on $y=0.5$. These comparison plots are presented in Fig. 9 (for $T=2.0$ ) and Fig. 10 (for $T=4.0$ ), respectively. A very nice agreement can be observed in the comparison plots. 

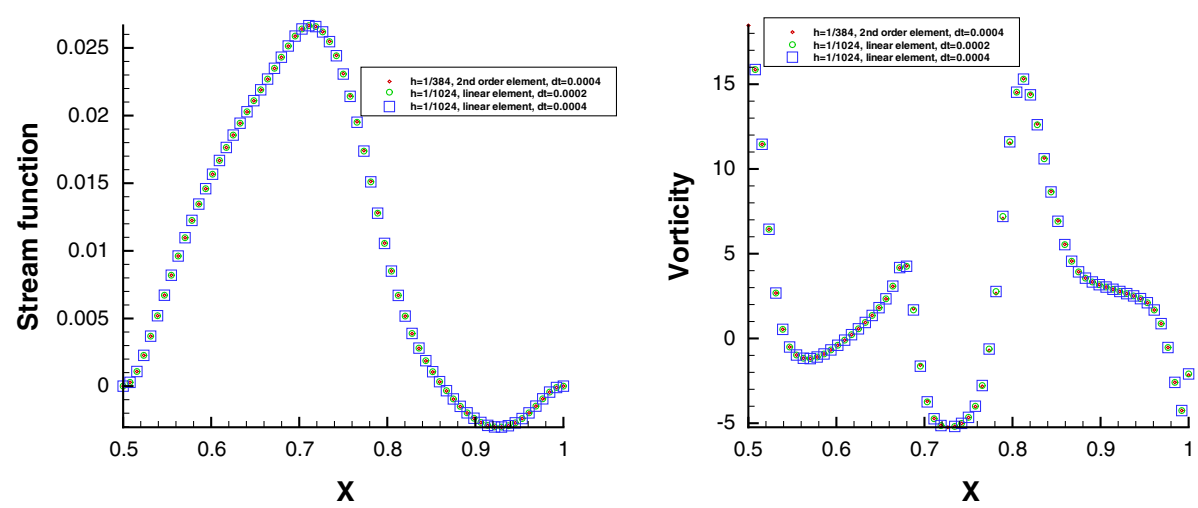

Fig. 9 Comparison of the triangle cavity flow at $y=0.5$ cut, for time $T=2.0$, using three resolutions. Left stream function comparison plot. Right vorticity comparison plot
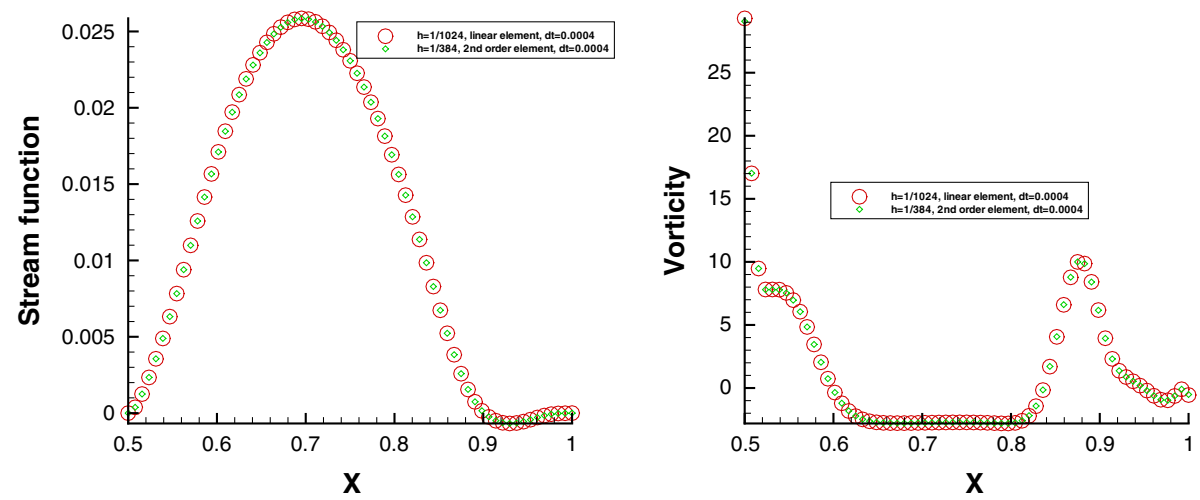

Fig. 10 Comparison of the triangle cavity flow at $y=0.5$ cut, for time $T=4.0$, using two resolutions. Left stream function comparison plot. Right vorticity comparison plot

\subsubsection{Example 2: Lid-Driven Triangle Cavity Flow with a Higher Reynolds Number $R e=10^{5}$}

More complicated structure transitions and vortex roll-up can be observed for the flow with a higher Reynolds number. In this subsection, we present the contour plots (including zoomed contours) of stream function and vorticity at two different time instants: $t=2.0$ and $t=4.0$, for the triangular cavity flow with $R e=10^{5}$, with the same physical set-up and initial data as in Sect. 3.1.1. To capture the boundary layer structure in a more precise way, we use the mesh parameter $h=\frac{1}{1536}$, and the time step size is taken as $\Delta t=2.0 E-4$. The contour plots of the stream function and vorticity profiles at time $t=2.0$ are given by Fig. 11, while the zoom contours around the right boundary $(x=1)$ and the oblique boundary $(x+y=1)$ are presented in Figs. 12, and 13, respectively. At a later time $t=4.0$, the corresponding contour plots and zoom contours could be observed in Figs. 14, 15 and 16. 

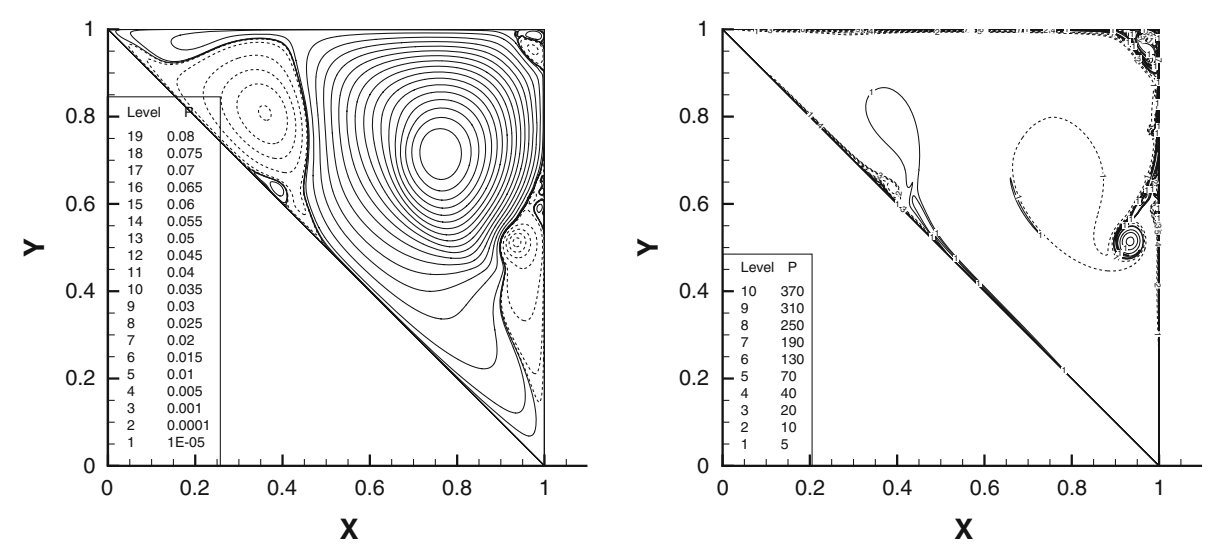

Fig. 11 The triangle cavity flow at time $t=2.0$, with a Reynolds number $R e=10^{5}$. Left stream function contours. Right vorticity contours
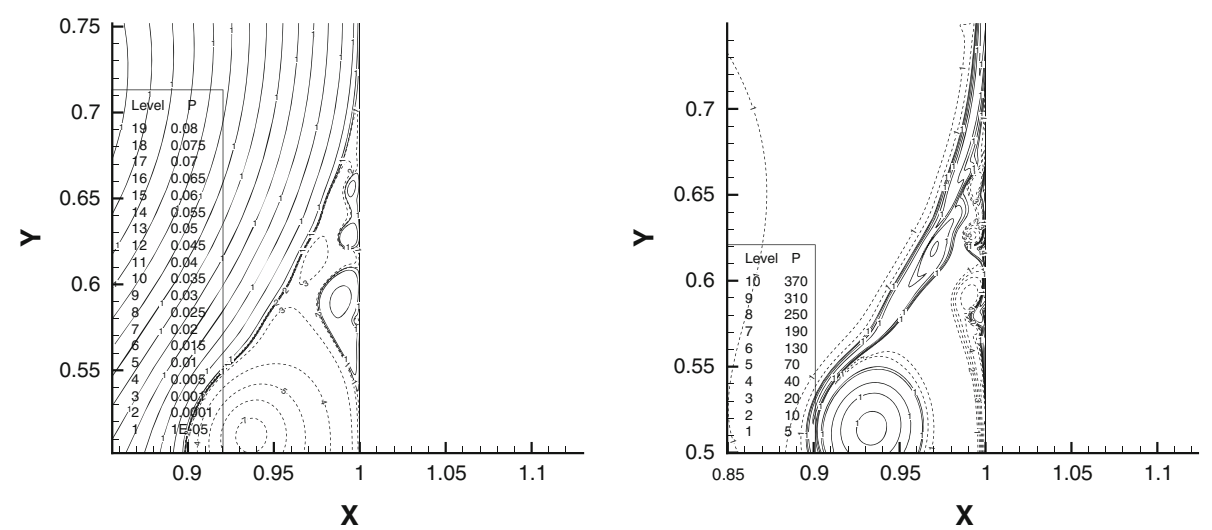

Fig. 12 Zoomed contours on the right boundary, for the triangle cavity flow at time $t=2.0$, with a Reynolds number $R e=10^{5}$. Left stream function contours. Right vorticity contours

\subsection{Numerical Simulation of the Impulsively Started Triangular Cavity Flow with \\ Reynolds Number $R e=10^{4}$}

In this set-up, a trivial initial data is taken: $\boldsymbol{u}_{0}(x, y) \equiv 0$, and a constant lid-driven velocity $u_{d} \equiv 1$ is imposed at the top boundary section $y=1$. In more detail, the no-penetration, no-slip boundary conditions are imposed on $\Gamma_{2}$ and a non-homogeneous Neumann boundary condition (with a constant slip velocity) is imposed on $\Gamma_{1}$ :

$$
\left.\frac{\partial \psi}{\partial \boldsymbol{n}}\right|_{\Gamma_{1}}=\left.\boldsymbol{u} \cdot \boldsymbol{\tau}\right|_{\Gamma_{1}} \equiv 1,\left.\quad \frac{\partial \psi}{\partial \boldsymbol{n}}\right|_{\Gamma_{2}}=0
$$

Due to the corner singularity (the horizontal velocity field $u$ becomes discontinuous at the upper-right corner $(1,1)$ ), the numerical simulation for this example is more challenging than that for the smoothly started flow. We present the contour plots of stream function and vorticity at two different time instants: $t=5.0$ (Fig. 17) and $t=15.0$ (Fig. 18), for the 

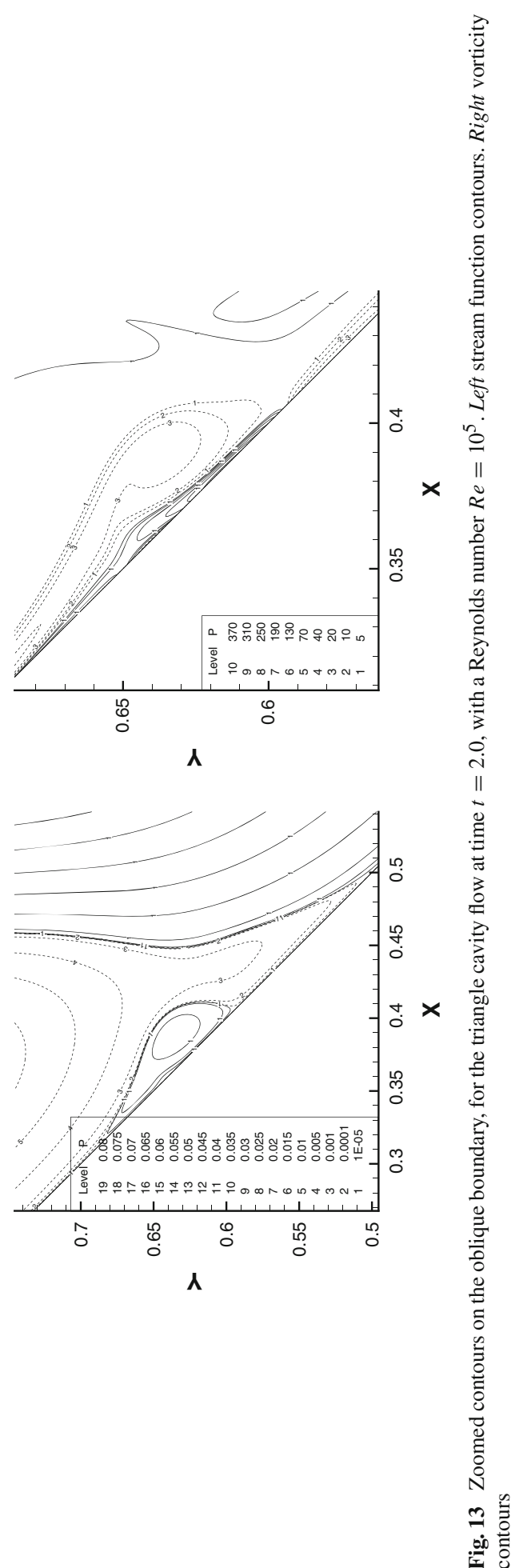

型 Springer 

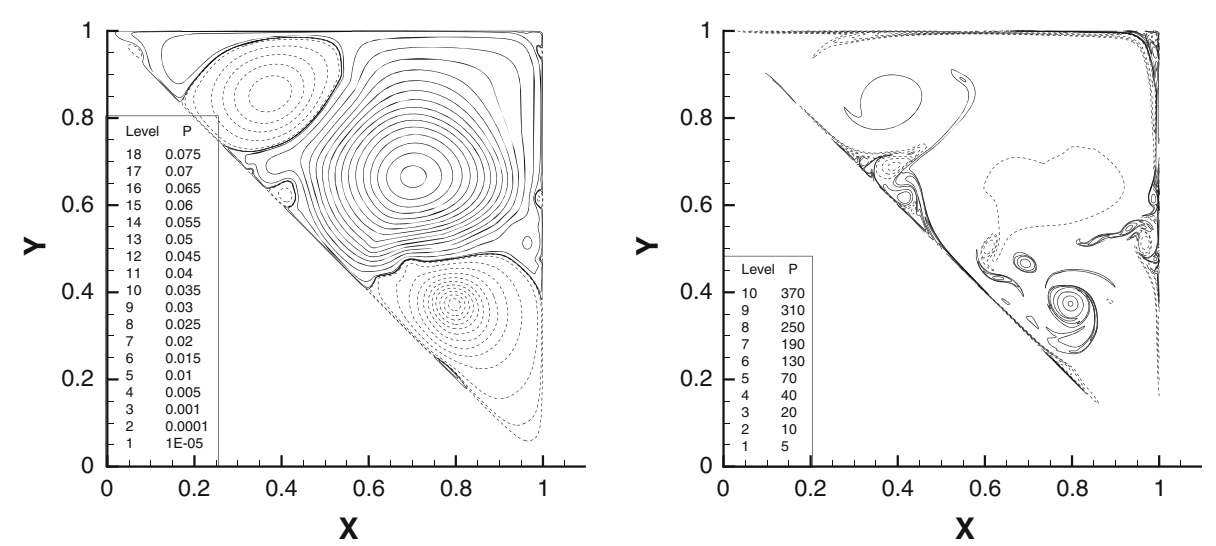

Fig. 14 The triangle cavity flow at time $t=4.0$, with a Reynolds number $R e=10^{5}$. Left stream function contours. Right vorticity contours
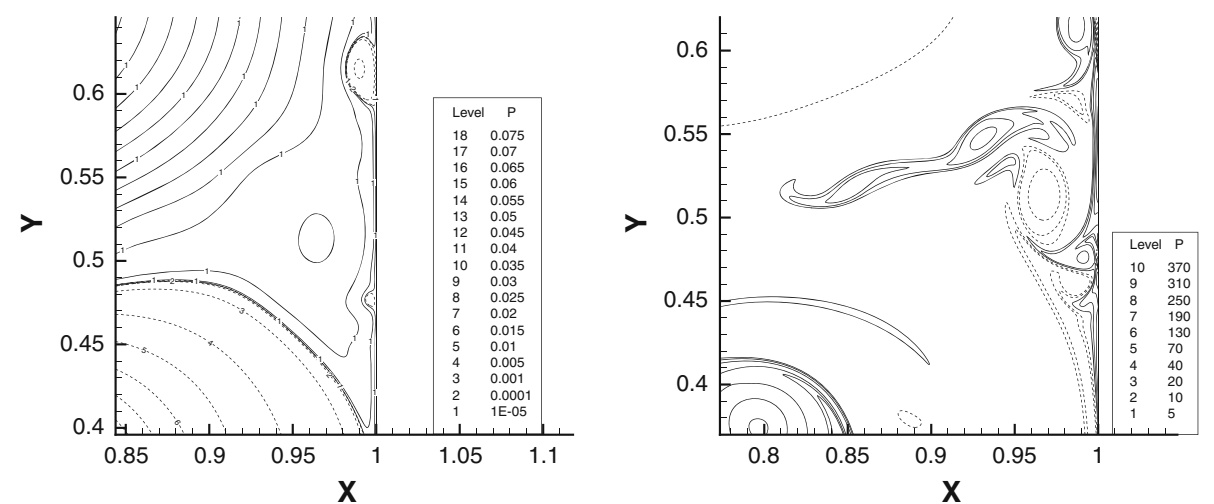

Fig. 15 Zoomed contours on the right boundary, for the triangle cavity flow at time $t=4.0$, with a Reynolds number $R e=10^{5}$. Left stream function contours. Right vorticity contours
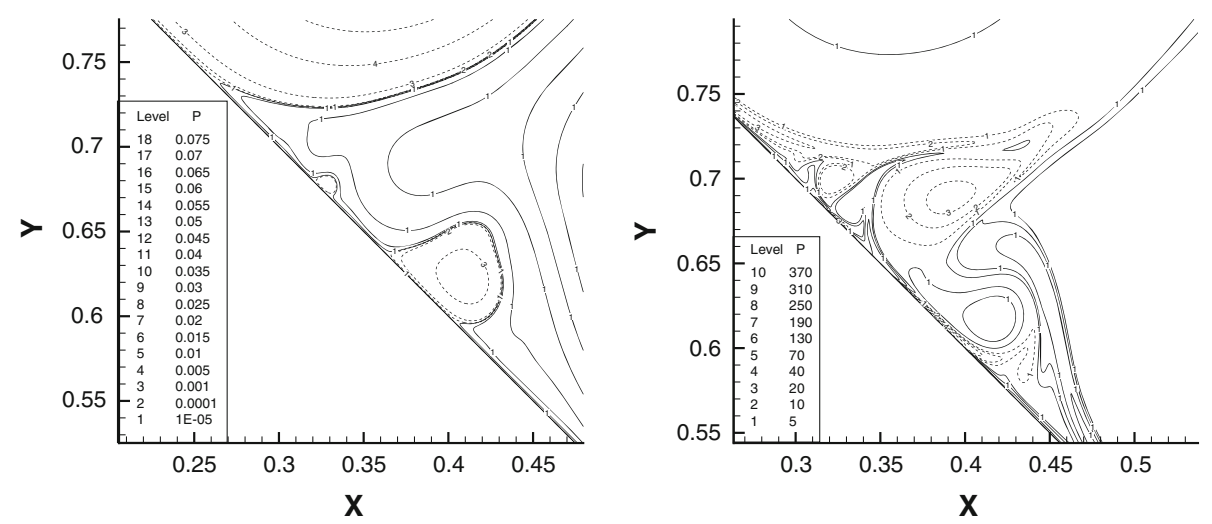

Fig. 16 Zoomed contours on the oblique boundary, for the triangle cavity flow at time $t=4.0$, with a Reynolds number $R e=10^{5}$. Left stream function contours. Right vorticity contours 

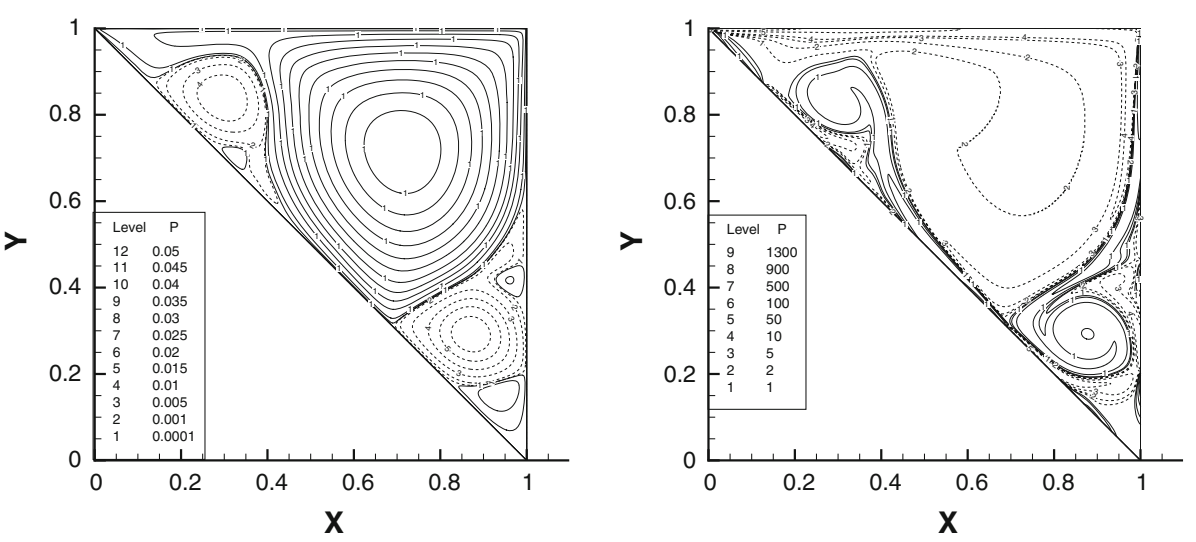

Fig. 17 The impulsively started triangle cavity flow at time $t=5.0$, with a Reynolds number $R e=10^{4}$. Left stream function contours. Right vorticity contours
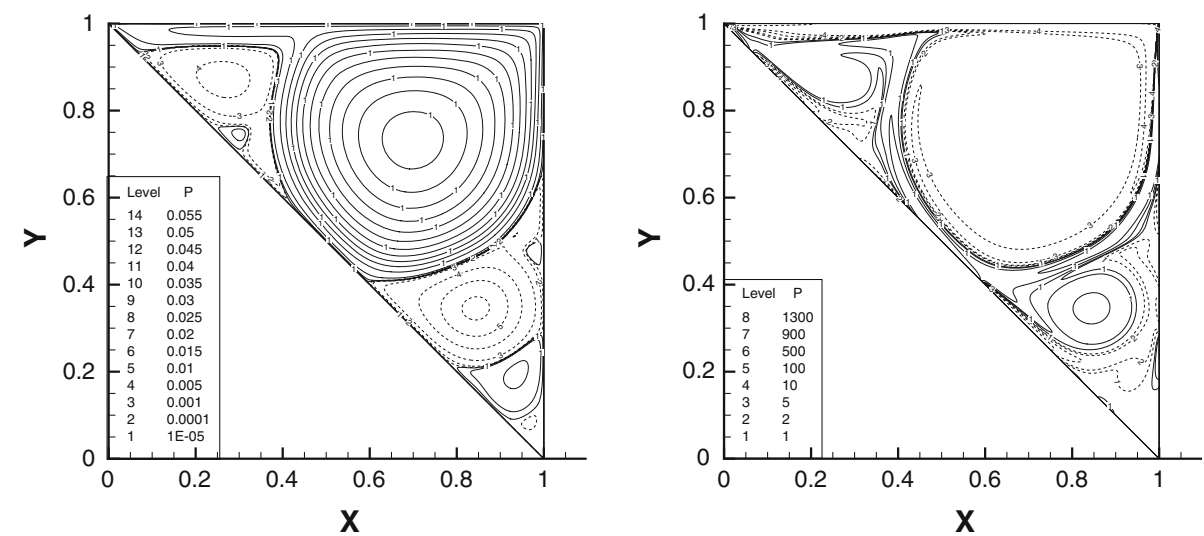

Fig. 18 The impulsively started triangle cavity flow at time $t=15.0$, with a Reynolds number $R e=10^{4}$. Left stream function contours. Right vorticity contours

impulsively started flow with $R e=10^{4}$, using the mesh size $h=\frac{1}{512}$. The time step size is taken as $\Delta t=1.0 E-03$ for $t \leq 100$, and $\Delta t=2.0 E-03$ for $t \geq 100$.

An obvious boundary layer separation could be observed along the oblique boundary section $x+y=1$, and its mechanism is very similar to that of the smoothly started flow. The details are skipped for simplicity of presentation.

In addition, to investigate the long time behavior of such a physical model, we present the time evolution (from $t=150.0$ to $t=300.0$ ) for the stream function and vorticity variables at the sample point $\left(\frac{7}{8}, \frac{13}{16}\right)$, a point close to the upper-right corner, also suggested by the reference [5] in the study of a square cavity flow, in Fig. 19). The periodic behavior is clearly observed for both variables.

Remark 3.4 All the finite element codes are generated by FEPG, pFEPG software ((Parallel) Finite Element Program Generator) [15]. The parallel computation are completed by Tianhe No. 1 in Tianjin, China. 

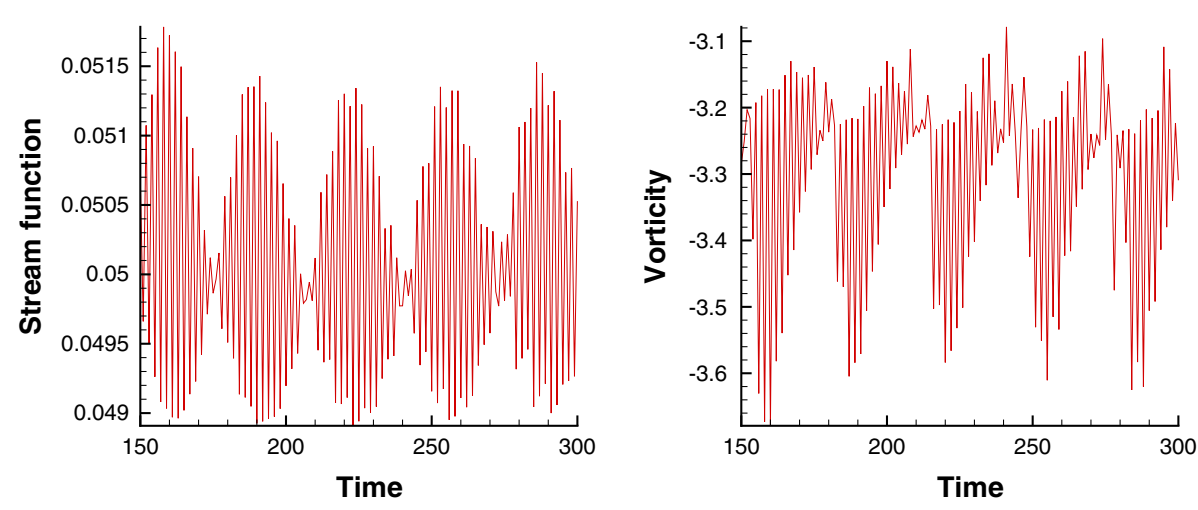

Fig. 19 The time evolution for the stream function and vorticity variables at the sample point $\left(\frac{7}{8}, \frac{13}{16}\right)$ for the impulsively started triangle cavity flow with $R e=10^{4}$. Left stream function plot. Right vorticity plot

Remark 3.5 The authors have posted the animated file of the numerical simulations online: http://202.113.29.3/ yhxue/index.html, with $R e=10^{5}$. Any interested reader can download and view the detailed process.

\section{Super-Convergence Error Estimate for the Semi-Discretization Scheme}

\subsection{A Preliminary Estimate of Super-Convergent Accuracy}

We denote $(\omega, \psi, \boldsymbol{u})$ as the exact solution to (1)-(3) and $\left(\omega_{h}, \psi_{h}, \boldsymbol{u}_{h}\right)$ as the simple finite element solution using linear element. The numerical errors of stream function, vorticity and velocity by the finite element method (7)-(10) are defined as follows

$$
\varepsilon=\omega-\omega_{h}, \quad \delta=\psi-\psi_{h}, \quad \tilde{\boldsymbol{u}}=\boldsymbol{u}-\boldsymbol{u}_{h}=(\tilde{u}, \tilde{v})=\nabla^{\perp} \delta .
$$

For the convenience of the error analysis in later section, we denote $P \omega$ as the $L^{2}$ projection of $\omega$ into the finite element space $X_{h}$ :

$$
(\omega-P \omega, \phi)=0, \quad \phi \in X_{h} .
$$

Furthermore, $\mathcal{I}_{h} \psi$ is defined as the interpolation of $\psi$ into $X_{0, h}$. The following results are available (see $[4,7])$

$$
\|\nabla(\omega-P \omega)\| \leq C h\|\omega\|_{2}, \quad\left\|\psi-\mathcal{I}_{h} \psi\right\|_{1, \infty} \leq C h\|\psi\|_{2, \infty} .
$$

We also introduce the projection and interpolation errors:

$$
\varepsilon_{h}=P \varepsilon=P \omega-\omega_{h}, \quad \delta_{h}=\mathcal{I}_{h} \delta=\mathcal{I}_{h} \psi-\psi_{h} .
$$

In addition, the following estimate is useful in the later convergence analysis:

$$
\left\|\partial_{t} \nabla\left(\delta-\delta_{h}\right)\right\|=\left\|\partial_{t} \nabla\left(\psi-\mathcal{I}_{h} \psi\right)\right\| \leq C h\left\|\partial_{t} \psi\right\|_{2} \leq C^{*} h,
$$

with $C^{*}$ only dependent on the exact solution $\psi$.

To obtain a super-convergence analysis for the simple finite element scheme, we need the following lemma. The proof is technical, though we refer the proof of a more special case in [42]. 
Fig. 20 A pair of neighboring triangles

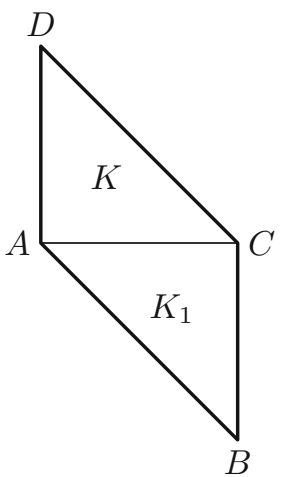

Lemma 4.1 For uniform triangulation $\mathcal{T}_{h}$ with right triangle elements where the sides of right angle are parallel to the coordinate axises, $\psi \in H_{0}^{3}(\Omega) \cap C^{0}(\Omega)$, then we have

$$
\int_{\Omega} \nabla \varepsilon_{h} \cdot \nabla\left(\psi-\mathcal{I}_{h} \psi\right) \leq C h^{2}\|\psi\|_{3}\left\|\varepsilon_{h}\right\|_{1}, \quad \forall \varepsilon_{h} \in X_{h} .
$$

where $C$ is the constant independent of $h$, and $X_{h}$ denotes the piecewise linear finite element space.

Proof We consider the two neighbor elements in the triangulation $\mathcal{T}_{h}$, see Fig. 20. Let $w=\psi-\mathcal{I}_{h} \psi, v=\varepsilon_{h}$ in (32), and $A=(0,0), B=(h,-h), C=(h, 0), D=(0, h)$. Thus in elements $K$ and $K_{1}, v_{x}$ has constant values. Therefore,

$$
\begin{aligned}
\int_{K \cup K_{1}} w_{x} v_{x} \mathrm{~d} x \mathrm{~d} y & =v_{x} \int_{K \cup K_{1}} w_{x} \mathrm{~d} x \mathrm{~d} y \\
& =v_{x}\left(\int_{l_{C D}} w \mathrm{~d} y-\int_{l_{A D}} w \mathrm{~d} y-\int_{l_{B A}} w \mathrm{~d} y+\int_{l_{B C}} w \mathrm{~d} y\right),
\end{aligned}
$$

where $l_{12}$ denote the line segment from point 1 to point 2 . In general, we define the following function on line $l$,

$$
F_{l}(P)=\frac{1}{2}\left(d^{2}(P, M)-\left(h_{l} / 2\right)^{2}\right),
$$

where $d(P, M)$ denotes the Euclidean distance from arbitrary point $P(x, y)$ to the mid-point $M\left(x_{M}, y_{M}\right)$ of $l$, and $h_{l}$ is the length of line segment $l$. It is clear that $F_{l}=0$ at the two end-points of the line segment.

On line segment $l_{A D}$, we have

$$
F_{l_{A D}}(P)=\frac{1}{2}\left((y-h / 2)^{2}-(h / 2)^{2}\right) \equiv f_{A D}(y),
$$

and

$$
\begin{aligned}
\int_{l_{A D}} w \mathrm{~d} y & =\int_{l_{A D}} w f_{A D}^{\prime \prime}(y) \mathrm{d} y=\left.w f_{A D}^{\prime}\right|_{0} ^{h}-\int_{l_{A D}} w_{y} f_{A D}^{\prime}(y) \mathrm{d} y \\
& =w f_{A D}^{\prime} l_{0}^{h}-\left.w_{y} f_{A D}\right|_{0} ^{h}+\int_{l_{A D}} w_{y y} f_{A D}(y) \mathrm{d} y .
\end{aligned}
$$


Assuming $\psi \in C^{0}$, so $\mathcal{I}_{h} \psi$ is equal to $\psi$ at the vertex, and we have

$$
\int_{l_{A D}} w \mathrm{~d} y=\int_{l_{A D}} w_{y y} f_{A D}(y) \mathrm{d} y .
$$

Similarly, on line segment $l_{B C}, F_{l_{B C}}(P)$ is given by

$$
F_{l_{B C}}(P)=\frac{1}{2}\left((y+h / 2)^{2}-(h / 2)^{2}\right) \equiv f_{B C}(y),
$$

and the following identity is valid

$$
\int_{l_{B C}} w \mathrm{~d} y=\int_{l_{B C}} w_{y y} f_{B C}(y) \mathrm{d} y .
$$

A combination of these estimates yields

$$
\begin{aligned}
\int_{l_{B C}} w \mathrm{~d} y-\int_{l_{A D}} w \mathrm{~d} y & =\int_{K \cup K_{1}} f_{l}(y) w_{y y \tau} \mathrm{d} \tau \mathrm{d} y, \\
\text { with } \quad f_{l}(y) & =\frac{1}{2}\left(\left(y-y_{M}\right)^{2}-\frac{h^{2}}{4}\right),
\end{aligned}
$$

where $\left(x_{M}, y_{M}\right)$ is located in the line segment between $(0, h / 2)$ and $(h,-h / 2), f_{l}(y)=0$ on $l_{B A}$ and $l_{C D}$, and $\tau$ is the line parameter from $C$ to $D$.

We can deal with other pair of line integrals on $l_{C D}$ and $l_{B A}$ in a similar way. On $l_{C D}, F_{l_{C D}}(P)$ turns out to be

$$
F_{l_{C D}}(P)=\frac{1}{2}\left(d^{2}\left(P, M_{l_{C D}}\right)-\left(\frac{h_{l_{C D}}}{2}\right)^{2}\right) \equiv f_{l_{C D}}(\tau),
$$

where $\tau$ is the line parameter from $C$ to $D$. Therefore,

$$
\int_{l_{C D}} w \mathrm{~d} y=\frac{1}{\sqrt{2}} \int_{l_{C D}} w \mathrm{~d} \tau=\frac{1}{\sqrt{2}} \int_{l_{C D}} w_{\tau \tau} f_{l_{C D}}(\tau) \mathrm{d} \tau,
$$

and the integral on $l_{B A}$ becomes

$$
\int_{l_{B A}} w \mathrm{~d} y=\frac{1}{\sqrt{2}} \int_{l_{B A}} w_{\tau \tau} f_{l_{B A}}(\tau) \mathrm{d} \tau .
$$

These two estimates in turn gives

$$
\int_{l_{C D}} w \mathrm{~d} y-\int_{l_{B A}} w \mathrm{~d} y=\frac{1}{\sqrt{2}} \int_{K \cup K_{1}} f_{l}(\tau) w_{\tau \tau y} \mathrm{~d} y \mathrm{~d} \tau .
$$

Substituting (34) and (35) into (33), we have

$$
\int_{K \cup K_{1}} v_{x} w_{x} \leq C h^{2}\|w\|_{3, K \cup K_{1}}\|v\|_{1, K \cup K_{1}} .
$$

Denote the boundary of $\partial \Omega_{x}$ which is parallel to the $x$ axis. So the left elements $K$ satisfy $K \cap \partial \Omega_{x} \neq \emptyset$. In these elements, $w_{x}=0$. So summing up the estimate on all the pairs and these elements, we obtain

$$
\begin{aligned}
\int_{\Omega} v_{x} w_{x} & =\sum \int_{K \cup K_{1}} v_{x} w_{x}+\sum_{K \cap \partial \Omega_{x} \neq \emptyset} \int_{K} v_{x} w_{x} \\
& \leq C h^{2}\|w\|_{3}\|v\|_{1} .
\end{aligned}
$$


The following inequality could be derived in a similar manner:

$$
\int_{\Omega} v_{y} w_{y} \leq C h^{2}\|w\|_{3}\|v\|_{1}
$$

Finally, we complete the proof by letting $v=\varepsilon_{h}, w=\psi-\mathcal{I}_{h} \psi$.

\subsection{Error Estimate and Convergence Analysis}

Before the statement of the super-convergence result, we need the following lemma in the nonlinear analysis.

Lemma 4.2 For any $2 \leq p<\infty$, we have

$$
\left\|\boldsymbol{u}_{h}\right\|_{L^{2}\left(0, T ; L^{\infty}\right)} \leq C\left(\|\nabla \psi\|_{L^{2}\left(0, T ; L^{\infty}\right)}+h^{-\frac{2}{p}}\|\varepsilon\|_{L^{2}\left(0, T ; L^{2}\right)}\right) .
$$

Proof By the work of Rannacher and Scott [45], we know that the Ritz projection $\Pi$ is stable in $W_{0}^{1, p}$ for $2 \leq p \leq \infty$, with $W_{0}^{1, p}=\left\{\phi \in L^{p}, \nabla \phi \in\left(L^{p}\right)^{2},\left.\phi\right|_{\partial \Omega}=0\right\}$ :

$$
\|\Pi \psi\|_{W^{1, p}} \leq C\|\psi\|_{W^{1, p}} .
$$

Then we get

$$
\begin{aligned}
\left\|\boldsymbol{u}_{h}\right\|_{L^{2}\left(0, T ; L^{\infty}\right)} & \leq\left\|\nabla^{\perp} \Pi \psi\right\|_{L^{2}\left(0, T ; L^{\infty}\right)}+\left\|\nabla^{\perp}\left(\Pi \psi-\psi_{h}\right)\right\|_{L^{2}\left(0, T ; L^{\infty}\right)} \\
& \leq C\|\psi\|_{L^{2}\left(0, T ; W^{1, \infty}\right)}+\left\|\nabla\left(\Pi \psi-\psi_{h}\right)\right\|_{L^{2}\left(0, T ; L^{\infty}\right)}
\end{aligned}
$$

Meanwhile, we define $\zeta \in H_{0}^{1}(\Omega)$ as the solution of

$$
\Delta \zeta=\varepsilon,\left.\quad \zeta\right|_{\partial \Omega}=0
$$

Since $\Omega$ is convex, the following elliptic regularity estimate is valid:

$$
\|\zeta\|_{H^{2}} \leq C\|\varepsilon\|
$$

The definition of the Ritz projection $\Pi$ indicates that

$$
\left(\nabla \Pi \delta, \nabla \phi_{h}\right)=\left(\nabla \delta, \nabla \phi_{h}\right)=-\left(\varepsilon, \phi_{h}\right)=\left(\nabla \zeta, \nabla \phi_{h}\right), \quad \forall \phi_{h} \in X_{0, h} .
$$

This shows that $\Pi \delta$ is the Ritz projection of $\zeta$. Therefore, we obtain the stability property of $\Pi$ :

$$
\|\Pi \delta\|_{W^{1, p}} \leq C\|\zeta\|_{W^{1, p}} .
$$

Combing this with (41) and applying the Sobolev embedding $H^{2} \rightarrow W^{1, p}$ for $1 \leq p<\infty$, we have

$$
\|\Pi \delta\|_{W^{1, p}} \leq C\|\varepsilon\| ;
$$

also see the detailed analysis in Brenner and Scott [4]. Moreover, we observe that $\Pi \delta=$ $\Pi \psi-\psi_{h}$ is in the finite element space $X_{0, h}$, so that the following inverse inequality could be applied:

$$
\left\|\nabla\left(\Pi \psi-\psi_{h}\right)\right\|_{L^{\infty}} \leq C h^{-\frac{2}{p}}\|\Pi \delta\|_{W^{1, p}} .
$$

Therefore, we arrive at

$$
\left\|\nabla\left(\Pi \psi-\psi_{h}\right)\right\|_{L^{2}\left(0, T ; L^{\infty}\right)} \leq C h^{-\frac{2}{p}}\|\varepsilon\|_{L^{2}\left(0, T ; L^{2}\right)} .
$$

Substituting this estimate into (39), we finish the proof of Lemma 4.2. 
The following theorem is the main theoretical result of this article.

Theorem 4.1 Suppose that $(\omega, \psi)$ is the solution of weak formulation (7)-(8) and $\left(\omega_{h}, \psi_{h}\right)$ is the finite element approximation (9)-(10). Under the condition in Lemma 4.1, we have the super-convergence error estimate for the linear finite element method:

$$
\begin{aligned}
& \left\|\boldsymbol{u}-\boldsymbol{u}_{h}\right\|_{\boldsymbol{L}^{\infty}\left((0, T] ; \boldsymbol{L}^{2}\right)}+\sqrt{v}\left\|\omega-\omega_{h}\right\|_{L^{2}\left((0, T] ; L^{2}\right)} \\
& \leq \hat{C} h\left(\|\psi\|_{L^{2}\left((0, T] ; H^{3}\right)}+\|\omega\|_{L^{2}\left((0, T] ; H^{2}\right)}\right),
\end{aligned}
$$

where $\hat{C}$ is dependent on the exact solution, $v$ and $T$, independent on $h$.

Proof First, an $L^{2}\left(0, T ; L^{\infty}\right)$ a-priori assumption for the numerical solution of the velocity is made. Such an a-priori assumption will be recovered by the error estimate, which will be given later.

An a-priori assumption for the numerical solution $\boldsymbol{u}_{h}$ of the velocity variable We assume a-priori that numerical solution $\boldsymbol{u}_{h}$ has the following $L^{2}\left(0, T ; L^{\infty}\right)$ bound:

$$
\left\|\boldsymbol{u}_{h}\right\|_{L^{2}\left(0, T ; L^{\infty}\right)} \leq \tilde{C} .
$$

Since both the exact solution and approximate solution satisfy (9)-(10), we have

$$
\begin{aligned}
\left(\phi, \partial_{t} \varepsilon\right)-\left(\nabla \phi,\left(\omega \boldsymbol{u}-\omega_{h} \boldsymbol{u}_{h}\right)\right) & =-v(\nabla \phi, \nabla \varepsilon), \quad \forall \phi \in X_{0, h}, \\
(\nabla \phi, \nabla \delta) & =-(\phi, \varepsilon), \quad \forall \phi \in X_{h} .
\end{aligned}
$$

Taking the temporal derivative of (50), and then setting $\phi=\delta_{h}$, we see that the first term in (49) turns out to be

$$
\begin{aligned}
\left(\delta_{h}, \partial_{t} \varepsilon\right) & =-\left(\nabla \delta_{h}, \nabla \partial_{t} \delta\right)=-\left(\nabla \delta_{h}, \nabla \partial_{t} \delta_{h}\right)-\left(\nabla \delta_{h}, \nabla \partial_{t}\left(\delta-\delta_{h}\right)\right) \\
& =-\frac{1}{2} \frac{d}{d t}\left\|\nabla \delta_{h}\right\|^{2}-\left(\nabla \delta_{h}, \nabla \partial_{t}\left(\delta-\delta_{h}\right)\right)
\end{aligned}
$$

The nonlinear inner product term in (49) becomes

$$
\begin{aligned}
\left(\nabla \delta_{h},\left(\omega \boldsymbol{u}-\omega_{h} \boldsymbol{u}_{h}\right)\right) & =\left(\nabla \delta_{h}, \varepsilon \boldsymbol{u}_{h}\right)+\left(\nabla \delta_{h}, \omega\left(\boldsymbol{u}-\boldsymbol{u}_{h}\right)\right) \\
& =\left(\nabla \delta_{h}, \varepsilon \boldsymbol{u}_{h}\right)+\left(\nabla \delta_{h}, \omega \nabla^{\perp}\left(\delta-\delta_{h}\right)\right) \\
& =\left(\nabla \delta_{h}, \varepsilon \boldsymbol{u}_{h}\right)+\left(\nabla \delta_{h}, \omega \nabla^{\perp}\left(\psi-\mathcal{I}_{h} \psi\right)\right) \\
& \leq C_{1}(t)\left\|\nabla \delta_{h}\right\|\left(\left\|\nabla\left(\psi-\mathcal{I}_{h} \psi\right)\right\|+\|\varepsilon\|\right),
\end{aligned}
$$

with

$$
C_{1}(t)=M(t)+\|\omega(t)\|_{L^{\infty}}, M(t)=\left\|\boldsymbol{u}_{h}(t)\right\|_{L^{\infty}} .
$$

Therefore, we can estimate (49) in the following way

$$
\begin{aligned}
\frac{d}{d t}\left\|\nabla \delta_{h}\right\|^{2} \leq & 2 v\left(\nabla \delta_{h}, \nabla \varepsilon\right)+C_{1}(t)\left\|\nabla \delta_{h}\right\| \cdot\left(\left\|\nabla\left(\psi-\mathcal{I}_{h} \psi\right)\right\|+\|\varepsilon\|\right) \\
& -2\left(\nabla \delta_{h}, \nabla \partial_{t}\left(\delta-\delta_{h}\right)\right) .
\end{aligned}
$$

The last term on the right side of (54) could be analyzed as follows:

$$
\begin{aligned}
-2\left(\nabla \delta_{h}, \nabla \partial_{t}\left(\delta-\delta_{h}\right)\right) & \leq 2\left\|\nabla \delta_{h}\right\| \cdot\left\|\nabla \partial_{t}\left(\delta-\delta_{h}\right)\right\| \leq 2 C^{*} h\left\|\nabla \delta_{h}\right\| \\
& \leq\left(C^{*}\right)^{2} h^{2}+\left\|\nabla \delta_{h}\right\|^{2}
\end{aligned}
$$


with the inequality (31) applied in the second step. To estimate the first term on the right side of (54), we take $\phi=\varepsilon_{h}$ in (50) and get

$$
\begin{aligned}
\left(\varepsilon_{h}, \varepsilon_{h}\right) & =\left(\varepsilon_{h}, \varepsilon\right)=-\left(\nabla \varepsilon_{h}, \nabla \delta\right) \\
& =-(\nabla \varepsilon, \nabla \delta)+\left(\nabla\left(\varepsilon-\varepsilon_{h}\right), \nabla \delta\right) \\
& =-\left(\nabla \delta_{h}, \nabla \varepsilon\right)-\left(\nabla \varepsilon, \nabla\left(\delta-\delta_{h}\right)\right)+\left(\nabla\left(\varepsilon-\varepsilon_{h}\right), \nabla \delta\right) \\
& =-\left(\nabla \delta_{h}, \nabla \varepsilon\right)+\left(\nabla \delta_{h}, \nabla\left(\varepsilon-\varepsilon_{h}\right)\right)-\left(\nabla \varepsilon_{h}, \nabla\left(\delta-\delta_{h}\right)\right) \\
& =-\left(\nabla \delta_{h}, \nabla \varepsilon\right)+\left(\nabla \delta_{h}, \nabla(\omega-P \omega)\right)-\left(\nabla \varepsilon_{h}, \nabla\left(\psi-\mathcal{I}_{h} \psi\right)\right)
\end{aligned}
$$

Its combination with (54) and (55) shows that

$$
\begin{aligned}
\frac{d}{d t}\left\|\nabla \delta_{h}\right\|^{2}+2 v\left\|\varepsilon_{h}\right\|^{2} \leq & C_{1}(t)\left\|\nabla \delta_{h}\right\|\left(\left\|\nabla\left(\psi-\mathcal{I}_{h} \psi\right)\right\|+v\|\nabla(\omega-P \omega)\|\right. \\
& \left.+\|\omega-P \omega\|+\left\|\varepsilon_{h}\right\|\right)-2 v\left(\nabla \varepsilon_{h}, \nabla\left(\psi-\mathcal{I}_{h} \psi\right)\right) \\
& +\left(C^{*}\right)^{2} h^{2}+\left\|\nabla \delta_{h}\right\|^{2} \\
\leq & C_{1}(t)\left\|\nabla \delta_{h}\right\|\left(\left\|\varepsilon_{h}\right\|+h\left(\|\psi\|_{H^{2}}+\|\omega\|_{H^{2}}\right)\right) \\
& -2 v\left(\nabla \varepsilon_{h}, \nabla\left(\psi-\mathcal{I}_{h} \psi\right)\right)+\left(C^{*}\right)^{2} h^{2}+\left\|\nabla \delta_{h}\right\|^{2} .
\end{aligned}
$$

Using the super-convergent accuracy result in Lemma 4.1, the following estimate is valid:

$$
v\left(\nabla \varepsilon_{h}, \nabla(\psi-\Pi \psi)\right) \leq C \nu h^{2}\left\|\varepsilon_{h}\right\|_{1} \cdot\|\psi\|_{3} \leq C \nu h\left\|\varepsilon_{h}\right\|_{0} \cdot\|\psi\|_{3},
$$

with the inverse inequality applied at the second step. An application of the Cauchy-Schwarz inequality indicates that

$$
\frac{d}{d t}\left\|\nabla \delta_{h}\right\|^{2}+v\left\|\varepsilon_{h}\right\|^{2} \leq\left(\frac{C_{1}^{2}(t)}{v}+1\right)\left\|\nabla \delta_{h}\right\|^{2}+C_{2} h^{2}\left(\|\psi\|_{3}^{2}+\|\omega\|_{2}^{2}+1\right),
$$

with $C_{1}(t)$ and $C_{2}$ independent of $h$.

Note that $C_{1}(t)$ may not have a bounded value at any time $t$. On the other hand, from the $L^{2}\left(0, T ; L^{\infty}\right)$ bound (48) of the numerical solution for the velocity, we conclude that $C_{1}(t)$ is $L^{2}$ integrable:

$$
\begin{aligned}
\int_{0}^{T} C_{1}^{2}(t) d t & \leq 2 \int_{0}^{T}\left(M^{2}(t)+\|\omega(t)\|_{L^{\infty}}^{2}\right) d t \\
& \leq C_{3}:=2\left(\tilde{C}^{2}+\left(C_{0}^{*}\right)^{2} T\right)
\end{aligned}
$$

with $C_{0}^{*}=\|\omega(t)\|_{L^{\infty}\left(0, T ; L^{\infty}\right)}$. Consequently, applying Gronwall inequality to (57) and setting the initial stream function as the interpolation: $\psi_{h}(0)=\mathcal{I}_{h} \psi(0)$, we arrive at

$$
\begin{aligned}
& \left\|\nabla \delta_{h}(t)\right\|^{2}+v \int_{0}^{t}\left\|\varepsilon_{h}(s)\right\|^{2} d s \\
& \leq C_{4} h^{2} \mathrm{e}^{\frac{C_{3}+T}{v}}\left(\|\psi\|_{L^{2}\left((0, T] ; H^{3}\right)}^{2}+\|\omega\|_{L^{2}\left((0, T] ; H^{2}\right)}^{2}\right) .
\end{aligned}
$$

This is equivalent to

$$
\begin{aligned}
& \left\|\nabla \delta_{h}\right\|_{L^{\infty}\left((0, T] ; L^{2}\right)}+\sqrt{v}\left\|\varepsilon_{h}\right\|_{L^{2}\left((0, T] ; L^{2}\right)} \leq \hat{C} h \\
& \text { with } \hat{C}=\sqrt{C_{4}} \mathrm{e}^{\frac{C_{3}+T}{2 v}}\left(\|\psi\|_{L^{2}\left((0, T] ; H^{3}\right)}+\|\omega\|_{L^{2}\left((0, T] ; H^{2}\right)}\right) .
\end{aligned}
$$

The $O(h)$ super-convergence (47) has been derived. 
Recovery of the a-priori assumption (48) By the numerical error definitions (28) and (30), we have

$$
\|\varepsilon(t)\| \leq\left\|\varepsilon_{h}(t)\right\|+\|\omega-P \omega\| \leq\left\|\varepsilon_{h}(t)\right\|+C h\|\omega(t)\|_{2}, \quad \forall t \geq 0 .
$$

Consequently, the $O(h)$ convergence result (60) for the $L^{2}\left(0, T ; L^{2}\right)$ norm of the vorticity variable results in

$$
\|\varepsilon\|_{L^{2}\left(0, T ; L^{2}\right)} \leq C\left(\frac{\hat{C}}{v^{1 / 2}}+1\right) h .
$$

Therefore, the a-priori assumption (48) is justified, with an application of Lemma 4.2:

$$
\begin{aligned}
\left\|\boldsymbol{u}_{h}\right\|_{L^{2}\left(0, T ; L^{\infty}\right)} \leq & C\left(\|\nabla \psi\|_{L^{2}\left(0, T ; L^{\infty}\right)}+h^{-\frac{1}{2}}\|\varepsilon\|_{L^{2}\left(0, T ; L^{2}\right)}\right) \\
& (\text { by taking } p=4 \text { in }(37)) \\
\leq & C\left(\|\boldsymbol{u}\|_{L^{2}\left(0, T ; L^{\infty}\right)}+\left(\frac{\hat{C}}{v^{1 / 2}}+1\right) h^{1 / 2}\right) \\
\leq & \tilde{C}=C C_{1}^{*}+1, \quad \text { with } C_{1}^{*}=\|\boldsymbol{u}\|_{L^{2}\left(0, T ; L^{\infty}\right)},
\end{aligned}
$$

under the condition that $h \leq \frac{v}{C^{2} \hat{C}^{2}}$. This completes the proof of Theorem 4.1.

Remark 4.1 To overcome the difficulty associated with the nonlinearity, we make an a-priori assumption (48) for the $L^{2}\left(0, T ; L^{\infty}\right)$ bound of the numerical velocity variable. In turn, an $O(h)$ convergence $(60)$ is derived for both the $L^{\infty}\left(0, T ; L^{2}\right)$ error of the velocity and the $L^{2}\left(0, T ; L^{2}\right)$ error of the vorticity. With this convergence result at hand, we could recover the a-priori $L^{2}\left(0, T ; L^{\infty}\right)$ assumption (48) for the velocity, with the help of Lemma 4.2 (by taking $p=4$ in (37)).

In fact, the $O(h)$ convergence (60) could be established in an alternate way, without making the a-priori assumption (48). By making use of Lemma 4.2 (with $p=4$ ), we are able to get an inequality analogous to (57):

$$
\begin{aligned}
& y^{\prime}(t) \leq \breve{C}\left(\frac{y^{2}}{h}+y+h^{2}\right), \quad \text { with } y(t)=\left\|\nabla \delta_{h}(t)\right\|^{2}+v \int_{0}^{t}\left\|\varepsilon_{h}(t)\right\|^{2} d \boldsymbol{x}, \\
& y(0)=O\left(h^{2}\right),
\end{aligned}
$$

in which $\breve{C}$ only depends on the exact solution and $v$, independent on $h$. In turn, a careful analysis for this ODE shows that an $O(h)$ convergence (60) is valid, over a fixed final time $T$. The details are skipped for the sake of brevity.

Remark 4.2 A careful calculation shows that, the external force term $f$ does not affect the convergence order. The reason is that the inner product $(f, \phi)$ is cancelled between the exact solution and the numerical solution, so that it will not appear in the numerical error equation.

Remark 4.3 As mentioned in the introduction, for a general triangulation $\mathcal{T}_{\langle}$of domain $\Omega$, a convergence order for the linear finite element has not been theoretically justified in the existing literature, due to the difficulty associated with the $L^{\infty}$ bound for the numerical solution; see the related discussions in [43]. In this paper, we have obtained the first order convergence under the assumptions in Lemma 4.1. As a result, this is the super-convergence result. 


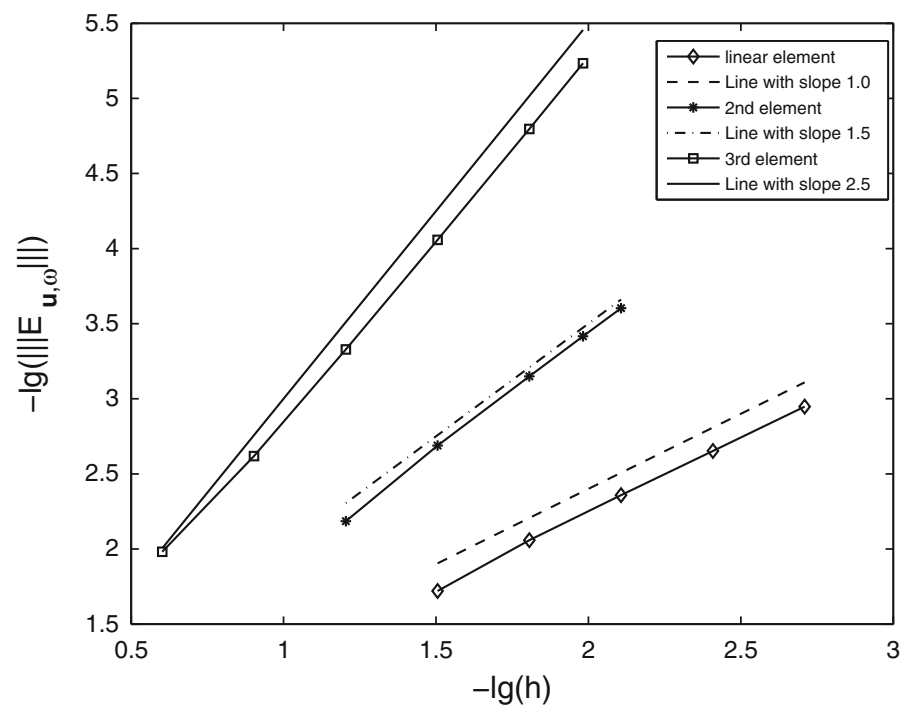

Fig. 21 Convergence orders for the simple finite element approximation in the energy norm (67), using the linear, 2rd and 3rd order elements, respectively. The time step: $\Delta t / h=0.64$ for the linear element, $\Delta t / h=1.28$ for the 2 nd order element, and $\Delta t / h=0.48$ for the 3 rd order element

\section{Accuracy Check}

In order to verify the super-convergence result, a numerical experiment is implemented over the trapezoid domain $\Omega$ with four edges: $x=0, y=0, x+y=2, y=1$. The exact stream function is given by the following smooth profile:

$$
\psi_{e}(x, y, t)=x^{2} y^{2}(y-1)^{2}(x+y-2)^{2} \cos (t) .
$$

The exact velocity and vorticity can be calculated based on the stream function above. In the test, the Reynolds number is taken to be $R e=10^{4}$ (i.e., viscosity parameter $v=10^{-4}$ ), and final time is given by $T=1.0$. The computation is carried out on the uniform triangulation of $\Omega$.

For the ease of exposition, we define the error as $E_{\boldsymbol{u}, \omega} \equiv\left(\boldsymbol{u}-\boldsymbol{u}_{h}, \omega-\omega_{h}\right)$, and also the energy norm as

$$
\left\|\left|E_{\boldsymbol{u}, \omega}\|\mid \equiv\| \boldsymbol{u}-\boldsymbol{u}_{h}\left\|_{\boldsymbol{L}^{\infty}\left((0, T] ; \boldsymbol{L}^{2}(\Omega)\right)}+\right\| \omega-\omega_{h} \|_{L^{2}\left((0, T] ; L^{2}(\Omega)\right)} .\right.\right.
$$

The classical RK4 is taken as the time stepping procedure for (9)-(10), and the numerical stability is assured if the stability condition (11) is satisfied. The convergence orders for the simple finite element scheme, using the linear, the 2nd and 3rd order elements, are displayed in Fig. 21. For the linear element with a uniform triangulation, a clear first order convergence rate is observed in the energy norm, and this numerical result matches the theoretical analysis stated in Theorem 4.1. For the $2 \mathrm{nd}(\mathrm{k}=2)$ and $3 \mathrm{rd}$ order $(\mathrm{k}=3)$ finite element approximations, the convergence orders are given by 1.5 and 2.5 in the energy norm, by a careful observation. The displayed results confirm the theoretical analysis stated in the earlier article [43]: one half less convergence order than the full order.

In other words, the following fact is confirmed by our numerical results: For the convergence in the energy norm, which combines the $L^{\infty}\left(0, T ; L^{2}\right)$ convergence for the velocity 


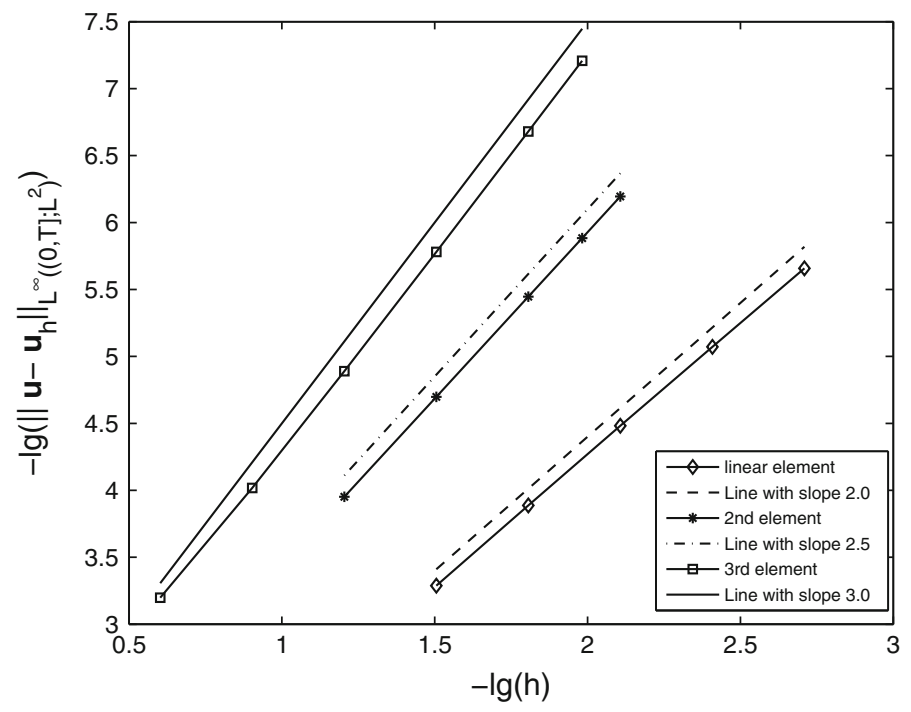

Fig. 22 Convergence orders for the simple finite element approximation in the $L^{\infty}\left(0, T ; L^{2}\right)$ norm of the velocity error, using the linear, $2 \mathrm{rd}$ and $3 \mathrm{rd}$ order elements, respectively. The time step set-up is the same as Fig. 21

and the $L^{2}\left(0, T ; L^{2}\right)$ convergence for the vorticity, the super-convergence is valid for the linear element scheme, while the 2 nd and the 3 rd order element do not have the super-convergent property.

Furthermore, a more subtle fact has been observed through our numerical experiments. If we only consider the $L^{\infty}\left(0, T ; L^{2}\right)$ convergence for the velocity and skip the $L^{2}\left(0, T ; L^{2}\right)$ convergence for the vorticity, all these elements do have super-convergence property. In more detail, the 2nd order element has a convergence order $=2.5$, and the 3 rd order element has a convergence order $=3$ and the linear element scheme has an even stronger super-convergence property, with convergence order=2. See the displayed numerical results in Fig. 22.

Conjecture 5.1 Suppose that $(\omega, \psi)$ is the solution of (7)-(8) and $\left(\omega_{h}, \psi_{h}\right)$ is the finite element approximation (9)-(10). Under the condition in Lemma 4.1, super-convergence for the $L^{\infty}\left(0, T ; L^{2}\right)$ error estimate of the velocity is valid for the simple finite element method, with any order of element:

$$
\begin{aligned}
& \left\|\boldsymbol{u}-\boldsymbol{u}_{h}\right\|_{\boldsymbol{L}_{\left((0, T] ; \boldsymbol{L}^{2}\right)}} \leq \hat{C} h^{2}, \quad \text { with } k=1, \text { linear element } \\
& \left\|\boldsymbol{u}-\boldsymbol{u}_{h}\right\|_{\boldsymbol{L}_{\left((0, T] ; \boldsymbol{L}^{2}\right)}} \leq \hat{C} h^{k}, \quad \text { with } k \text {-th order element }
\end{aligned}
$$

where $\hat{C}$ is dependent on the exact solution, $v$ and $T$, independent on $h$.

A theoretical justification for this conjecture is still open. We may work on this problem in the future.

\section{Concluding Remarks}

We apply a simple finite element scheme for 2-D incompressible fluid to carry out a detailed numerical study of boundary layer separation of a triangular cavity flow with Reynolds 
numbers $R e=10^{4}$ and $R e=10^{5}$. The numerical efficiency is accomplished by a fully explicit RK4 time discretization, with only two Poisson/Poisson-like solvers needed at each time step/stage. Compared to many existing finite element fluid solver, this numerical approach enables us to capture the detailed structures of high Reynolds number fluid, in particular for the boundary layer separation process, due to its Stokes-solver free nature.

Meanwhile, it is observed that a convergence analysis for this numerical scheme using linear elements has not been theoretically justified in the existing literature, due to the difficulty to get an $L^{\infty}$ bound for the numerical solution. In this paper, we provide a super-convergence analysis for this simple finite element numerical scheme, using linear elements over a uniform triangulation with right triangles. The subtle difficulties associated with the nonlinear terms are overcome in an appropriate way. By making use of the super-convergent property of a uniform triangulation, we are able to improve the convergence order of the energy norm, from $O\left(h^{1 / 2}\right)$ to $O(h)$, under an a-priori $L^{2}\left(0, T ; L^{\infty}\right)$ bound of the numerical solution $\boldsymbol{u}_{h}$. In turn, we apply a $W^{1,4}$ analysis for the finite element Poisson solver, and its combination with a modified inverse inequality implies an $O\left(h^{1 / 2}\right)$ estimate for the velocity numerical error function in the $L^{2}\left(0, T ; L^{\infty}\right)$ norm. This process justifies the a-priori $L^{2}\left(0, T ; L^{\infty}\right)$ assumption so that the super-convergence result is proven.

Moreover, an even stronger super-convergence property has been observed in our extensive numerical experiments, if only the $L^{\infty}\left(0, T ; L^{2}\right)$ error of the velocity variable is considered. A conjecture is also formulated, and its theoretical justification is open to any interested researchers.

Acknowledgments The authors greatly appreciate many helpful discussions with Wenbin Chen, Sigal Gottlieb, Yuan Liu and Chi-Wang Shu, in particular for their insightful suggestion and comments. This work is supported in part by the NSF DMS-1115420 NSF DMS-1418689 (C. Wang), NSFC 11271281 (C. Wang), NSFC 11171168 (Y. Xue) and the fund by China Scholarship Council (Y. Xue). Y. Xue thanks University of Massachusetts Dartmouth, for support during his visit. C. Wang also thanks Shanghai Center for Mathematical Sciences and Shanghai Key Laboratory for Contemporary Applied Mathematics, Fudan University, for support during his visit.

\section{References}

1. Auteri, F., Parolini, N., Quartapelle, L.: Numerical investigation on the stability of singular driven cavity flow. J. Comput. Phys. 183, 1-25 (2002)

2. Barragy, E., Carey, G.F.: Stream function-vorticity driven cavity solution using $p$ finite elements. Comput. Fluids 26(5), 453-468 (1997)

3. Boffi, D., Brezzi, F., Fortin, M.: Mixed Finite Element Methods and Applications. Springer, Berlin (2013)

4. Brenner, S., Scott, L.: The Mathematical Theory of Finite Element Methods, 3rd edn. Springer, Berlin (2010)

5. Bruneau, C.-H., Saad, M.: The 2D lid-driven cavity problem revisited. Comput. Fluids 35, 326-348 (2006)

6. Chorin, A.J., Marsden, J.E.: A Mathematical Introduction to Fluid Mechanics. Springer, Berlin (1997)

7. Ciarlet, P.: The Finite Element Method for Elliptic Problems. North-Holland, Amsterdam (1978)

8. Cockburn, B., Kanschat, G., Schötzau, D.: A locally conservative LDG method for the incompressible Navier-Stokes equations. Math. Comput. 74, 1067-1095 (2005)

9. Coupez, T., Hachem, E.: Solution of high-Reynolds incompressible flow with stabilized finite element and adaptive anisotropic meshing. Comput. Methods Appl. Mech. Eng. 267, 65-85 (2013)

10. Di, Y., Li, R., Tang, T., Zhang, P.: Moving mesh finite element methods for the incompressible NavierStokes equations. SIAM J. Sci. Comput. 26, 1036-1056 (2005)

11. E, W., Liu, J.-G.: Essentially compact schemes for unsteady viscous incompressible flows. J. Comput. Phys. 126, 122-138 (1996)

12. E, W., Liu, J.-G.: Vorticity boundary condition and related issues for finite difference schemes. J. Comput. Phys. 124, 368-382 (1996) 
13. Erturk, E., Corke, T., Gokcol, C.: Numerical solutions of 2D steady incompressible driven cavity flow at high Reynolds numbers. Int. J. Numer. Methods Fluids 48, 747-774 (2005)

14. Erturk, E., Gokcol, O.: Fine grid numerical solutions of triangular cavity flow. Eur. Phys. J. Appl. Phys. 38, 97-105 (2007)

15. China Fegensoft software company. http://www.fegensoft.com.cn

16. Center for Applied Scientific Computing of Lawrence Livermore National Lab. https://computation-rnd. 1lnl.gov/linear_solvers/software.php

17. Gargano, F., Sammartino, M., Sciacca, V.: High Reynolds number Navier-Stokes solutions and boundary layer separation induced by a rectilinear vortex. Comput. Fluids 52, 73-91 (2011)

18. Ge, L., Sotiropoulos, F.: A numerical method for solving the 3D unsteady incompressible Navier-Stokes equations in curvilinear domains with complex immersed boundaries. J. Comput. Phys. 225, 1782-1809 (2007)

19. George, A., Huang, L.C., Tang, W., Wu, Y.: Numerical simulation of unsteady incompressible flow ( $R e \leq 9500)$ on the curvilinear half-staggered mesh. SIAM J. Sci. Comput. 21(6), 2331-2351 (2000)

20. Ghia, U., Ghia, K., Shin, C.: High-Re solutions for incompressible flow using the Navier-Stokes equations and a multigrid method. J. Comput. Phys 48, 387-411 (1982)

21. Ghil, M., Liu, J.-G., Wang, C., Wang, S.: Boundary-layer separation and adverse pressure gradient for 2-D viscous incompressible flow. Phys. D 197, 149-173 (2004)

22. Ghil, M., Ma, T., Wang, S.: Structure of 2-D incompressible flows with the Dirichlet boundary conditions. Discrete Contin. Dyn. Syst. B 1, 29-41 (2001)

23. Ghil, M., Ma, T., Wang, S.: Structural bifurcation of 2-D nondivergent flows with Dirichlet boundary condition: applications to boundary-layer separation. SIAM J. Appl. Math. 65(5), 1576-1596 (2005)

24. Girault, V., Raviart, P.: Finite Element Method for Navier-Stokes Equations: Theory and Algorithms. Springer, Berlin (1986)

25. Girault, V., Riviére, B., Wheeler, M.F.: A discontinuous Galerkin method with nonoverlapping domain decomposition for the Stokes and Navier-Stokes problems. Math. Comput. 74, 53-84 (2005)

26. Goldstein, S.: Modern Developments in Fluid Fynamics. Dover Publications, New York (1965)

27. Gonzalez, L.M., Ahmed, M., Kuhnen, J.: Three-dimensional flow instability in a lid-driven isosceles triangular cavity. J. Fluid Mech. 675, 369-396 (2011)

28. Hachem, E., Rivaux, B., Kloczko, T., Digonnet, H., Coupez, T.: Stabilized finite element method for incompressible flows with high Reynolds number. J. Comput. Phys. 229, 8643-8665 (2010)

29. He, Y.: Euler implicit/explicit iterative scheme for the stationary Navier-Stokes equations. Numer. Math. 123, 67-96 (2013)

30. Heywood, J., Rannacher, R.: Finite element approximation of the nonstationary Navier-Stokes problem. I. Regularity of solutions and second-order error estimates for spatial discretization. SIAM J. Numer. Anal. 19, 275-310 (1982)

31. Holdeman, J.T.: A Hermite finite element for incompressible fluid flow. Int. J. Numer. Methods Fluids 64, 376-408 (2010)

32. Huang, Y., Liu, J.-G., Wang, W.: A generalized MAC scheme on curvilinear domains. SIAM J. Sci. Comput. 35(5), 953-986 (2013)

33. Jagannathan, A., Mohan, R., Dhanak, M.: A spectral method for the triangular cavity flow. Comput. Fluids 95, 40-48 (2014)

34. Johnston, H., Liu, J.-G.: A finite difference scheme for incompressible flow based on local pressure boundary conditions. J. Comput. Phys. 180, 120-154 (2002)

35. Johnston, H., Liu, J.-G.: Accurate, stable and efficient Navier-Stokes solvers based on explicit treatment of the pressure term. J. Comput. Phys. 199, 221-259 (2004)

36. Kalita, J.C., Sen, S.: The biharmonic approach for unsteady flow past an impulsively started circular cylinder. Commun. Comput. Phys. 12, 1163-1182 (2012)

37. Koumoutsakost, P., Leonard, A.: High-resolution simulations of the flow around an impulsively started cylinder using vortex methods. J. Fluid Mech. 296, 1-38 (1995)

38. Lai, M.-J., Wenston, P.: Bivariate splines for fluid flows. Comput. Fluids 33, 1047-1073 (2004)

39. Layton, W., Lee, H., Peterson, J.: A defect-correction method for the incompressible Navier-Stokes equations. Appl. Math. Comput. 129, 1-19 (2002)

40. Li, M., Tang, T.: Steady viscous flow in a triangular cavity by efficient numerical techniques. Comput. Math. Appl. 31(10), 55-65 (1996)

41. Li, Z., Wang, C.: A fast finite difference method for solving Navier-Stokes equations on irregular domains. Commun. Math. Sci. 1(1), 181-197 (2003)

42. Lin, Q., Yan, N.: Structure and Analysis for Efficient Finite Element Methods. Publishers of Hebei University, Baoding (1996). (in Chinese) 
43. Liu, J.-G., E, W.: Simple finite element methods in vorticity formulation for incompressible flows. Math. Comput. 70(234), 579-593 (2000)

44. Oleinik, O.A., Samokhin, V.N.: Mathematical Models in Boundary Layer Theory. Chapman and Hall, London (1999)

45. Rannacher, R., Scott, R.: Some optimal error estimates for piecewise linear finite element approximations. Math. Comput. 38, 437-445 (1982)

46. Rhebergen, S., Cockburn, B., van der Vegt, J.J.W.: A space-time discontinuous Galerkin method for the incompressible Navier-Stokes equations. J. Comput. Phys. 233, 339-358 (2013)

47. Ribbens, C.J., Watson, L.T., Wang, C.-Y.: Steady viscous flow in a triangular cavity. J. Comput. Phys. 112(1), 173-181 (1994)

48. Shahbazi, K., Fischer, P.F., Ethier, C.R.: A high-order discontinuous Galerkin method for the unsteady incompressible Navier-Stokes equations. J. Comput. Phys. 222, 391-407 (2007)

49. Wang, K., Wong, Y.S.: Error correction method for Navier-Stokes equations at high Reynolds numbers. J. Sci. Comput. 255, 245-265 (2013)

50. Yan, N.: Superconvergence Analysis and a Posteriori Error Estimation in Finite Element Methods. Science Press, China (2005) 